\title{
From Box Algebra to Interval Temporal Logic
}

\author{
Hanna Klaudel \\ IBISC, University of Evry, Université Paris-Saclay \\ 91025, Evry, France \\ hanna.klaudel@ibisc.univ-evry.fr
}

\author{
Zhenhua Duan \\ ICTT and ISN Laboratory, Xidian University \\ Xi'an, 710071, P.R. China \\ zhhduan@mail.xidian.edu.cn
}

\author{
Maciej Koutny \\ School of Computing, Newcastle University \\ Newcastle upon Tyne, NE4 5TG, United Kingdom \\ maciej.koutny@ncl.ac.uk
}

\author{
Ben Moszkowski \\ School of Computing, Newcastle University \\ Newcastle upon Tyne, NE4 5TG, United Kingdom \\ benmos63@gmail.com
}

\begin{abstract}
In this paper, we further develop a recently introduced semantic link between temporal logics and Petri nets. We focus on two specific formalisms, Interval Temporal Logic (ITL) and Box Algebra (BA), which are closely related by their compositional approach to constructing system descriptions. The overall goal of our investigation is to translate Petri nets into behaviourally equivalent logical formulas. As a result, the analysis of system properties can be carried out using either of the two formalisms, exploiting their respective strengths and powerful tool support.
\end{abstract}

The contribution of this paper is twofold. First, we extend the existing translation from BA to ITL, by removing restrictions concerning the way control flow of concurrent system is modelled, and by allowing a fully general synchronisation operator. Second, we strengthen the notion of equivalence between a Petri net and the corresponding logical formula by proving such an equivalence at the level of transition-based executions of Petri nets rather than just by looking at their labels. We also show that the complexity of the proposed translation compares favourably with the complexity of the translation from BA expressions to Petri nets.

Keywords: Interval Temporal Logic, Petri nets, box algebra, composition, semantics, general synchronisation, step sequence, equivalence 


\section{Introduction}

Temporal logics $[1,2,3,4]$ and Petri nets $[5,6]$ are generally regarded as fundamentally different approaches to the specification and analysis of concurrent systems. The former allow one to specify both the system designs and correctness requirements within a single logical framework, and the verification of correctness can be done by checking the satisfaction of formulas, or model checking. In contrast, Petri nets are an automata-inspired model with semantics based on actions and local states which allows one to capture causal relationships in systems' behaviour. As a result, verification of behavioural properties can be carried out using invariant techniques [7], based on the graph structure of nets, or model checking techniques, based on partial order semantics [8,9].

To establish a semantic link between logics and Petri nets, we focused in [10] on two specific formalisms, Interval Temporal Logic (ITL) [11, 12] and Box Algebra (BA) [13], which are closely related by the their compositional approaches to constructing system descriptions. In particular, in both ITL and BA the control flow of a system is specified by means of commonly used programming operators, such as sequence, choice, parallelism, and iteration. The synchronisation between concurrently executed subsystems is, however, achieved in different ways and therefore needs to be suitably handled.

Box Algebra [13], and its precursor Petri Box Calculus [14], provided a generic process-algebraic syntax together with a compositional translation to a class of Petri nets called boxes. This generic algebra has several concrete incarnations, including CCS [15] and TCSP [16]. BA can also readily yield a compositional semantics for an imperative concurrent programming language [17]. It has also been extended to handle, e.g., stochastic aspects of concurrent systems [18].

In contrast to point-based temporal logics (e.g., [2,3]), where computations are described in terms of successive system states, interval temporal logics aim at capturing the evolution of a system referring to its behaviour over time intervals. As pointed out in [19], the concept of time intervals is both naturally appealing and intuitive, and has been of significant interest to several disciplines, including Philosophy, Linguistics, Artificial Intelligence, and Computer Science. For example, interval temporal logics allow one to easily capture concepts relating to action duration or shared-variable concurrency (e.g., [20]). The preface [4] of a journal special issue on interval temporal logics identified, in particular, two categories of temporal logics formalisms for intervals: (i) modal formalisms such as HS-logics [21] and CDT [22] with interval-based variables and capable of expressing Allen's relations [23] (recent developments concerning model checking HS-logics include [24, 25, 26, 27, 28]); and (ii) Interval Temporal Logic (ITL) of [29, 30, 12] together with its variations and adaptations which use state-based variables and the sequential composition 'chop' operator to a significant extent. In this paper we focus on ITL which has influenced several research groups and projects, as well as IEEE standard 1647 ([31]). It is not unusual for researchers to develop their own variants of ITL, often with quite different names. The Duration Calculus [32], a prominent real-time variant of ITL, is the best example. A recent instance of this is Multi-Lane Spatial Logic [33], which is intended for modeling road traffic (e.g, for computer-based assistance of car drivers). ITL lends itself to execution, and can support abstract specifications and concrete implementations in the same notation, with refinement mappings between. Moreover, timings of executions can be easily derived by considering interval lengths. 


\subsection{About this paper}

In [10], we proved the correctness of a translation from a submodel of BA to semantically equivalent ITL formulas. The submodel we considered disallowed the nesting of the parallel composition operator. Moreover, synchronisation was binary. In the paper [34], we demonstrated how to extend the submodel of [10] to handle data variables.

In this paper, which is a full and extended version of a conference publication [35], we provide a syntax-driven translation for the core BA [13] syntax comprising parallel composition, sequence, choice, synchronisation, and iteration but without considering data variables (incorporating these using the technique introduced in [34] would be straightforward but, at the same time, would obscure the key constructs introduced in the present paper). Crucially, we relax the syntactical constraints preventing the use of the parallel composition outside the topmost level of process expressions. Moreover, we consider a fully general synchronisation operator. Finally, we strengthen the notion of equivalence between a BA net and the corresponding ITL formula by proving such an equivalence at the level of transition-based executions of Petri nets, rather than just by taking their labels. It is worth noting that for the purposes of this paper, i.e., the translation of the basic BA, we only need to use propositional ITL (PITL).

To formalise a semantic link between BA and ITL, for every logical formula we introduce a step sequence semantics which records variables changing their values at each computational step. This allows us to compare its behaviour with the step sequences of the corresponding Petri net, and to conclude their full equivalence in the main result (Theorem 4.3). In essence, the latter states that any property which can be captured within the step sequence model can be analysed using either of the two equivalent representations, i.e., a Petri net or a logical formula.

\subsection{Paper organisation}

The paper is organised as follows. In the next section, we recall the basic notions of Box Algebra. We start with the definition of box expressions, which are then used to compositionally construct box nets. We also recall a number of results demonstrating how the execution semantics of a composite box can be derived from the execution semantics of its components. In Section 3, we similarly recall the relevant fragment of Interval Temporal Logic, and present some basic semantic properties of ITL formulas. Section 4 is central to the whole paper as it contains a formal translation from box expressions to ITL formulas as well as the proof of a semantic equivalence between box expressions and the corresponding formulas. The following section presents examples of this translation, and Section 6 briefly discusses future work.

\subsection{An introductory example}

In order to outline the main ideas behind the proposed solution, we will now consider a small fragment of an application involving two concurrent processes, a server and a client, following a simple interactive protocol where: $(i)$ the client process sends a query to a database server, awaits the answer, and then carries out a local update; and ( $i i)$ the server process non-deterministically chooses between 
handling a client request or performing its own local update:

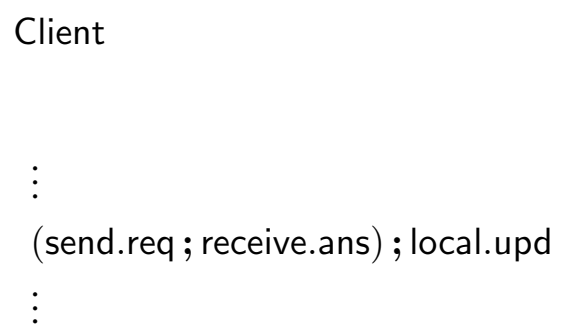

Client

From the above fragment of pseudo-code we can extract in a straightforward way the following Box Algebra representation, where ; and $\square$ respectively denote sequential composition and nondeterministic choice:

$$
\text { Client }=(\text { s.req;r.ans }) \text {; l.upd } \quad \text { Server }=(r . r e q ; s . a n s) \square \text { l.upd } .
$$

The box semantics of the above expressions is obtained compositionally, by first associating basic boxes (single-transition Petri nets) with atomic box expressions, and then by applying Box Algebra compositions rules. In general, Petri nets are directed graphs with two kind of nodes: places (local states) represented by circles, and transitions (actions) represented by squares or rectangles. The arcs connect nodes of different types and indicate direct relationships between places and transitions. In the box nets, additionally, circles labelled with e are entry places, circles labelled with i are internal places, and circles labelled with $\mathrm{x}$ are exit places, reflecting their intended role in net composition.

The successive construction stages of the box semantics for Server

$$
N_{\text {Server }}=N_{(\text {r.req } ; \text { s.ans }) \square l . u p d}=\left(N_{\text {r.req }} ; N_{\text {s.ans }}\right) \square N_{\text {l.upd }}
$$

are shown in Figure 1. First, two basic boxes $N_{\text {r.req }}$ and $N_{s . \text { ans }}$ (the two Petri nets in Figure 1(a)) are composed sequentially by gluing the exit (or final) place of $N_{r . r e q}$ with the entry (or initial) place of $N_{\text {s.ans. }}$. The resulting box $N_{\text {r.req }}$ s.ans (the Petri net in Figure 1(b)) is then composed with the basic box $N_{\text {l.upd }}$ by applying the non-deterministic choice operation, which glues together their entry places as well as their exit places (the final Petri net is shown in Figure 1(c)).

The net $N_{\text {Server }}$ we have just constructed provides the graph-theoretic (static) representation of the Server process. To obtain its dynamic (behavioural) representation, we need in addition to specify the initial marking (or state) of Server, from which one can explore all possible execution paths by following the standard transition firing rules. In the case of a box net, this is simply done by inserting a single token in each of its entry places (in this case, just one place). The initial marking can then be used to investigate the dynamic behaviour of Server, which is basically composed of two execution paths, both ending at a final marking in which the only exit place contains a token. One such path executes r.req followed by s.ans, and the other executes a single transition l.upd.

The states of the Server process are all the markings reachable from the initial one, and possible executions are represented by sequences of executed transitions (or sets of transitions, called steps). 


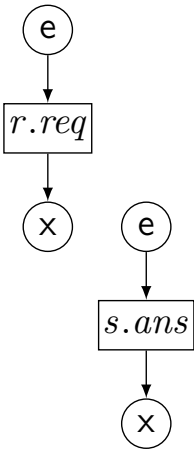

(a)

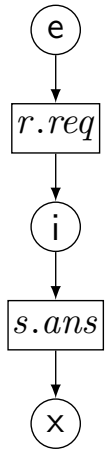

(b)

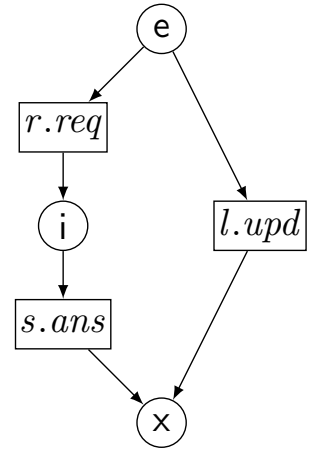

(c)

Figure 1. Compositional derivation of the box $N_{\text {Server }}=N_{(r . r e q ; s . a n s)}$ l.upd (a) basic boxes representing $r$.req and s.ans; (b) sequential composition representing $r . r e q ; s . a n s$; and (c) non-deterministic choice representing Server. Note that the entry and exit places are respectively labelled by e and $\mathrm{x}$; moreover, i identifies the internal places. All transitions are labelled by the corresponding atomic expressions occurring in the Server process.

In the ITL context, however, a state is a mapping which assigns values to a set of (Boolean) variables, and possible executions are represented by sequences of such assignments, called intervals. Therefore, we need a method to bridge the gap between these two different ways of representing the states and executions of concurrent systems. The solution we adopt and develop in this paper is to associate with each transition $t$ of $N_{\text {Server }}$ a distinct Boolean variable, also denoted by $t$, and then to represent each firing of transition $t$ by flipping the value of variable $t$; otherwise, the value of variable $t$ is kept unchanged. With this idea in mind, the box expression Server can be captured by the following ITL formula:

$$
\operatorname{itl}(\text { Server })=(\mathrm{fs}(\text { r.req } \mid \text { s.ans, l.upd }) ; \mathrm{fs}(\text { s.ans } \mid \text { r.req, l.upd })) \quad \vee \quad \mathrm{fs}(\text { l.upd } \mid \text { r.req, s.ans }) .
$$

where ; is the ITL "chop" operator ${ }^{1}$ (sequential composition) and a formula of the form $\mathrm{fs}(v \mid w, u)$ means that the values of the two variables $w$ and $u$ are kept unchanged while the value of $v$ can be flipped once within the interval over which the formula is evaluated (if $v$ is never flipped, the interval must be infinite). The execution path of $N_{\text {Server }}$ in which transition $r$.req is followed by s.ans can then be matched by a corresponding interval satisfying itl(Server) in which the variables $r . r e q$ and s.ans flip their values, as shown below:

\begin{tabular}{r|rrr} 
executed transitions & & r.req & \multicolumn{2}{c}{ s.ans } \\
\hline r.req & 0 & 1 & 1 \\
s.ans & 0 & 0 & 1 \\
l.upd & 0 & 0 & 0
\end{tabular}

\footnotetext{
${ }^{1}$ Subsequently adopted, e.g., by the Duration Calculus [32].
} 
Having seen how Server $=(r . r e q ; s . a n s) \square$ l.upd can be represented by an ITL formula, one might attempt to do exactly the same for a concurrent composition of both processes:

$$
\begin{aligned}
& ((\mathrm{fs}(\text { s.req } \mid \ldots) ; \mathrm{fs}(\text {. ans } \mid \ldots)) \quad ; \quad \mathrm{fs}(\text { l.upd } \mid \ldots)) \\
& \wedge \\
& ((\mathrm{fs}(\text { r.req } \mid \ldots) ; \mathrm{fs}(\text { s.ans } \mid \ldots)) \quad \vee \quad \mathrm{fs}(\text { l.upd } \mid \ldots))
\end{aligned}
$$

Such a naive translation exhibits two serious problems. The first is that it does not provide any means to synchronise the communication actions between the two processes. We address this issue by identifying some of the variables in different processes, such as s.req and r.req. The other problem is quite an opposite one, namely, the formula specifies that two different local updates must happen simultaneously as they are represented by the same ITL Boolean variable l.upd. We address this problem by introducing two different variables which are allowed to flip their values independently of each other. Before illustrating how both solutions work, we will complete the presentation of the net model for the example system.

The whole synchronised system comprising a client and a server is captured by the following box expression, where $\|$ is the parallel composition operator, and sco (for scoping) is an operator specifying all interprocess synchronisations [13]. For example, s.req, r.req $\mapsto$ req means that the simultaneously executed actions s.req and r.req can be synchronised, and replaced by a single new action req, while l.upd $\mapsto$ upd indicates that each action $l . u p d$ is simply maintained, after being renamed. Here is the resulting system expressed in Box Algebra, where the upper line represents server and the lower receiver:

$$
\text { System }=\left(\begin{array}{c}
(\text { s.req } ; \text { r.ans }) ; \text { l.upd } \\
\| \\
(\text { r.req } ; \text { s.ans }) \square l . u p d
\end{array}\right) \operatorname{sco}\left\{\begin{aligned}
\text { s.req, r.req } & \mapsto \text { req } \\
\text { s.ans, r.ans } & \mapsto \text { ans } \\
\text { l.upd } & \mapsto \text { upd }
\end{aligned}\right\}
$$

The box $N_{\text {System }}$ generated from the above expression is depicted in Figure 2. A crucial point is that during its generation each action (and then the corresponding transition) is made unique by annotating it with the path $(s)$ reflecting its position in the parse tree of the System expression. (Note that the construction stages shown in Figure 1 would also be amended to include appropriate annotations.) For example, $r . r e q_{\|_{R}} \square_{L} ;{ }_{L}$ means that $r$.req is reachable in the parse tree through the following path: from the root, labelled with $\|$, go to its right child $(R)$ corresponding to the second operand, labelled with $\square$, and from there visit its left child $(L)$ corresponding to the first operand, labelled with ; and, in turn, from here visit the left child $(L)$ corresponding to the first operand, which is the node labelled with r.req.

Thanks to annotating the individual actions with unique syntax paths, one can avoid unwanted synchronisations of independent actions, and then obtain an ITL translation of the whole synchronised system expression:

$$
\begin{aligned}
\operatorname{itl}(\text { System })= & ((\mathrm{fs}(\xi \mid \alpha, \gamma) ; \mathrm{fs}(\alpha \mid \xi, \gamma)) \quad ; \quad \mathrm{fs}(\gamma \mid \xi, \alpha)) \\
& \wedge \\
& ((\mathrm{fs}(\xi \mid \alpha, \delta) ; \mathrm{fs}(\alpha \mid \xi, \delta)) \quad \vee \quad \mathrm{fs}(\delta \mid \xi, \alpha))
\end{aligned}
$$




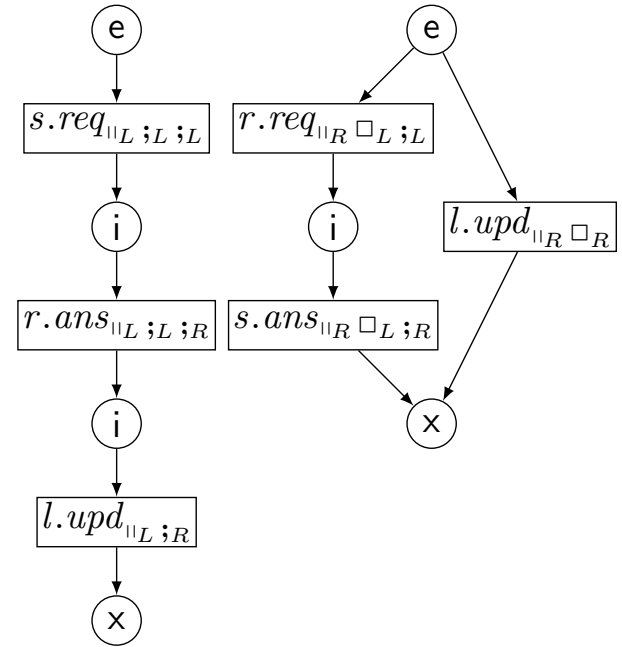

(a)

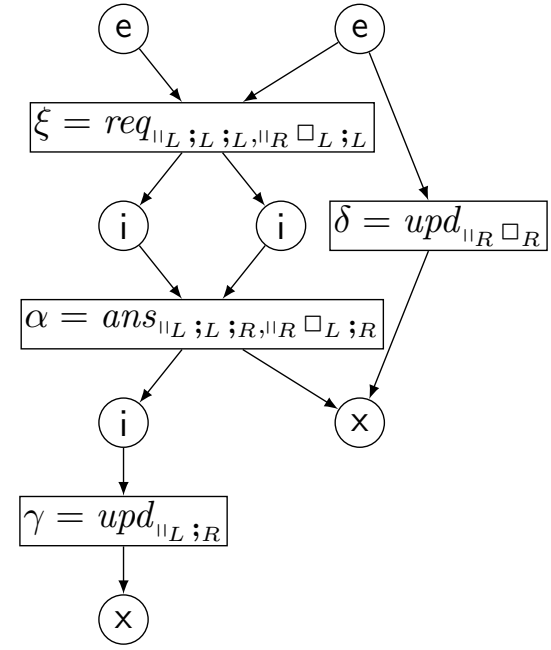

(b)

Figure 2. Boxes of Client $\|$ Server (a), and System (b).

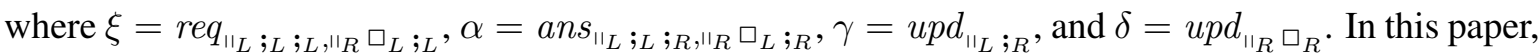
we show that the executions of the box shown in Figure 2 are consistent with the intervals satisfying the ITL formula itl(System) as, for example, in the following execution scenario:

\begin{tabular}{r|rrrr} 
executed transitions & & \multicolumn{2}{|c}{$\alpha$} & \multicolumn{2}{c}{$\gamma$} \\
\hline$\xi$ & 0 & 1 & 1 & 1 \\
$\alpha$ & 0 & 0 & 1 & 1 \\
$\gamma$ & 0 & 0 & 0 & 1 \\
$\delta$ & 0 & 0 & 0 & 0
\end{tabular}

\subsection{Notation}

The concatenation operator for sequences will be denoted by ".". For a finite sequence $\delta$ and a set of sequences $\Delta, \delta \cdot \Delta=\left\{\delta \cdot \delta^{\prime} \mid \delta^{\prime} \in \Delta\right\}$, and for a set of finite sequences $\Delta$ and a set of sequences $\Delta^{\prime}$, $\Delta \cdot \Delta^{\prime}=\left\{\delta \cdot \delta^{\prime} \mid \delta \in \Delta \wedge \delta^{\prime} \in \Delta^{\prime}\right\}$. The $k$-th element of a sequence $\delta$ will be denoted by $\delta_{(k)}$ and its length by $|\delta|(|\delta|=\omega$ if $\delta$ is infinite). We will use the following notations involving sequences of empty sets: $\varnothing^{*}=\{\epsilon, \varnothing, \varnothing \varnothing, \ldots\}$ is the infinite set of all finite sequences of empty sets, and $\varnothing^{\omega}=\{\varnothing \varnothing \ldots\}$ is the set containing a single infinite sequence of empty sets. 


\section{Box Algebra}

Let $\mathcal{A}$ be a finite set of atomic actions. A synchronisation relation $\rho$ is a finite set of tuples of actions $\left(a_{1}, \ldots, a_{n}, a\right)$ with $n \geq 1$. Intuitively, $a_{1}, \ldots, a_{n}$ represent $n$ concurrent actions which can be synchronised to yield a single composite action with the label $a$. To reflect this intuition, we will often denote $\left(a_{1}, \ldots, a_{n}, a\right)$ by $a_{1} \ldots a_{n} \mapsto a$.

The syntax given below defines two kinds of box expressions, namely, non-synchronised expressions $E$ capturing the control flow in a concurrent system, and synchronised expressions $F$ (below $a$ is an action and $\rho$ a synchronisation relation):

$$
\begin{array}{ll}
E & :=\operatorname{stop}|a| E ; E|E \square E| E \| E \mid \llbracket E \circledast E \circledast E \rrbracket \\
F & ::=E \operatorname{sco} \rho
\end{array}
$$

The intuition behind the above syntax is that: $(i)$ stop denotes a blocked process; $(i i) a$ denotes a process which can execute an action $a \in \mathcal{A}$ and terminate; (iii) $E ; E^{\prime}$ denotes sequential composition; (iv) $E \square E^{\prime}$ denotes non-deterministic choice composition; $(v) E \| E^{\prime}$ denotes parallel composition; $(v i) \llbracket E \circledast E^{\prime} \circledast E^{\prime \prime} \rrbracket$ denotes a loop with an initial part $E$, iterated part $E^{\prime}$, and terminal part $E^{\prime \prime}$; and (vii) $E$ sco $\rho$ denotes scoping, which first creates all the synchronisations specified by $\rho$ and then deletes all the actions of $E$.

\subsection{Box nets}

The semantics of box expressions is given through a mapping into Petri nets called boxes.

A box is a tuple $N=(P, T, F, \ell)$, where: $(i) P$ and $T$ are disjoint finite sets of respectively places (representing local states) and transitions (representing actions); (ii) $F \subseteq(P \times T) \cup(T \times P)$ is a flow relation; and $($ iii $) \ell$ is a labelling function for places and transitions such that $\ell(p) \in\{\mathrm{e}, \mathrm{i}, \mathrm{x}\}$ associates an entry, internal, or exit status with every place $p$, and an atomic action $\ell(t) \in \mathcal{A}$, with every transition $t$.

The sets of entry, internal and exit places of $N$ are given respectively by $N^{\mathrm{e}}=\ell^{-1}(\mathrm{e}), N^{\mathrm{i}}=$ $\ell^{-1}(\mathrm{i})$, and $N^{\mathrm{x}}=\ell^{-1}(\mathrm{x})$. Moreover, we set $N^{\mathrm{ei}}=N^{\mathrm{e}} \cup N^{\mathrm{i}}$ and $N^{\mathrm{ix}}=N^{\mathrm{i}} \cup N^{\mathrm{x}}$, and will use $N^{\mathrm{P}}$, $N^{\mathrm{T}}$, and $N^{\mathrm{F}}$ to respectively denote the places, transitions, and the flow relation (arcs) of $N$. We also adopt the standard rules about representing nets as directed graphs.

\subsection{Semantics of box nets}

The global states of a box $N$ are called markings, each marking being a mapping $M$ assigning a nonnegative integer to every place in $N^{\mathrm{P}}$. The default initial (or entry) and final (or exit) markings of $N$, denoted respectively by $M_{N}^{\mathrm{e}}$ and $M_{N}^{\mathrm{x}}$, are defined, for every $p \in N^{\mathrm{P}}$, as follows:

$$
M_{N}^{\mathrm{e}}(p)=\left\{\begin{array}{ll}
1 & \text { if } p \in N^{\mathrm{e}} \\
0 & \text { otherwise }
\end{array} \quad \text { and } \quad M_{N}^{\mathrm{x}}(p)= \begin{cases}1 & \text { if } p \in N^{\times} \\
0 & \text { otherwise }\end{cases}\right.
$$

The change of a marking of $N$ results from a simultaneous execution of a (possibly empty) set of transitions, called a step. Formally, a step of $N$ is any set of transitions $U \subseteq N^{\top}$. It is enabled at a 
marking $M$ if, for every place $p \in N^{\mathrm{P}}$ :

$$
M(p) \geq|\{t \in U \mid(p, t) \in F\}| .
$$

We denote this by $M[U\rangle$. An enabled step $U$ can be executed leading to a marking $M^{\prime}$ given, for every place $p \in N^{\mathrm{P}}$, by:

$$
M^{\prime}(p)=M(p)-|\{t \in U \mid(p, t) \in F\}|+|\{t \in U \mid(t, p) \in F\}| .
$$

We denote this by $M[U\rangle M^{\prime}$.

The semantics of $N$ is given through its infinite step sequences starting from the default initial marking $M_{N}^{\mathrm{e}}$. In addition, we single out a set of finite step sequences which lead from the default initial marking $M_{N}^{\mathrm{e}}$ to the default final marking $M_{N}^{\mathrm{x}}$. Intuitively, each such step sequence will be interpreted as a successfully terminating execution of $N$.

A step sequence of $N$ is an infinite sequence $\theta=U_{1} U_{2} \ldots$ of steps such that there exist markings $M_{1}, M_{2}, \ldots$ of $N$ satisfying

$$
M_{N}^{\mathrm{e}}\left[U_{1}\right\rangle M_{1} M_{1}\left[U_{2}\right\rangle M_{2} M_{2}\left[U_{3}\right\rangle M_{3} \quad \ldots
$$

Moreover, we define a terminating step sequence of $N$ as a finite sequence $\theta=U_{1} \ldots U_{m}(m \geq 0)$ of steps such that there exist markings $M_{1}, \ldots, M_{m-1}$ of $N$ satisfying

$$
M_{N}^{\mathrm{e}}\left[U_{1}\right\rangle M_{1} M_{1}\left[U_{2}\right\rangle M_{2} M_{2}\left[U_{3}\right\rangle M_{3} \quad \ldots \quad M_{m-1}\left[U_{m}\right\rangle M_{N}^{\times} .
$$

The sets of (infinite) step sequences and terminating step sequences of $N$ are respectively denoted by $\operatorname{infsts}(N)$ and finsts $(N) .^{2}$

\subsection{Composite boxes}

The above definition of a box net is too general for our purposes, as we are interested in nets derived compositionally from box expressions. The labelling of places will provide the necessary device for composing boxes along the entry and exit interfaces, i.e., the sets of entry places $N^{\mathrm{e}}$ and exit places $N^{\times}$.

The nets we are going to construct will have a very specific form of places and transitions, making it easier to establish connections between boxes and ITL formulas. Intuitively, we will use the syntax of a non-synchronised box expression $E$ to construct concrete places and transitions of the corresponding box $N$ by embedding paths from the root of the parse tree of $E$ in the definitions of places and transitions. In what follows, finite sequences in the set

$$
\Pi=\left\{;_{L}, ;_{R}, \square_{L}, \square_{R},\left\|_{L},\right\|_{R}, \circledast_{L}, \circledast_{M}, \circledast_{R}\right\}^{*}
$$

will be called syntax paths. Note that symbols appearing in syntax paths correspond to the arguments of operators used in box expressions (with ' $L$ ' indicating the left operand, $\circledast_{M}$ the middle operand,

\footnotetext{
${ }^{2}$ Note that a deadlock corresponds to a step sequence $\theta \in \operatorname{infsts}(N)$ such that it can be decomposed as $\theta=\theta^{\prime} \cdot \varnothing \varnothing \ldots$ and $\left\{\theta^{\prime \prime} \mid \theta^{\prime} \cdot \theta^{\prime \prime} \in \operatorname{infsts}(N)\right\}=\varnothing^{\omega}$.
} 
etc). For two syntax paths, $\pi_{1}$ and $\pi_{2}$, we use $\pi_{1} \mid \pi_{2}$ to denote that $\left\{\pi_{1}, \pi_{2}\right\}=\left\{\pi \cdot\left\|_{L} \cdot \pi^{\prime}, \pi \cdot\right\|_{R} \cdot \pi^{\prime \prime}\right\}$, for some $\pi, \pi^{\prime}$, and $\pi^{\prime \prime}$. Intuitively, two actions of a non-synchronised box expression are concurrent iff their positions $\pi_{1}$ and $\pi_{2}$ in the syntax (or parse) tree are such that $\pi_{1} \mid \pi_{2}$.

The form of each place in composite boxes will be $p_{Z}$, where $p \in\{\mathrm{e}, \mathrm{i}, \mathrm{x}\}$ and $Z \subseteq \Pi \cdot\{\mathrm{e}, \mathrm{x}\}$, while each transition will be of the form $a_{W}$, where $a \in \mathcal{A}$ and $W \subseteq \Pi$. Moreover, for brevity, the sets $Z$ and $W$ will be written as comma-separated lists without brackets.

For a syntax path $\pi \in \Pi$ and a transition $a_{W}$, we denote $\pi \cdot a_{W}=a_{\pi \cdot W}$. This prefix notation extends in the usual way to sets of transitions and sequences of sets of transitions as well as (sets of) places.

The specific form of places and transitions, together with the systematic way in which boxes are manipulated below, will mean that for a compositional box $N=(P, T, F, \ell)$ it will be the case that, for all $p_{Z} \in P$ and $a_{W} \in T, \ell\left(p_{Z}\right)=p$ and $\ell\left(a_{W}\right)=a$, as well as:

$$
\begin{aligned}
& \left(p_{Z}, a_{W}\right) \in F \quad \Longleftrightarrow \exists \pi \in W: \pi \cdot \mathrm{e} \in Z \\
& \left(a_{W}, p_{Z}\right) \in F \quad \Longleftrightarrow \exists \pi \in W: \pi \cdot \mathrm{x} \in Z .
\end{aligned}
$$

Thus both the flow relation and labelling function are implicit, and we will represent such a box simply by $N=(P, T)$.

We will now present a systematic way of constructing composite boxes from box expressions, i.e., for each constant expression we define a box and, for each operator used in box expressions, a corresponding operator on boxes.

Constants With the blocking expression stop and a single-action expression $a \in \mathcal{A}$, we respectively associate the following boxes:

$$
N_{\text {stop }}=\left(\left\{\mathrm{e}_{\mathrm{e}}, \mathrm{x}_{\mathrm{x}}\right\}, \varnothing\right) \text { and } N_{a}=\left(\left\{\mathrm{e}_{\mathrm{e}}, \mathrm{x}_{\mathrm{x}}\right\},\left\{a_{\epsilon}\right\}\right) .
$$

Their diagrams are depicted in Figure 3 with labels shown inside the nodes. $N_{\text {stop }}$ consists of one entry place, one exit place, and nothing else. $N_{a}$ contains, in addition, a single transition. The way it is connected with the two places is determined by the formula (1). For example, $\left(\mathrm{e}_{\mathrm{e}}, a_{\epsilon}\right) \in F$ since $\pi=\epsilon$ is an annotation of $a_{\epsilon}$ and $\pi \cdot \mathrm{e}=\mathrm{e}$ is an annotation of $\mathrm{e}_{\mathrm{e}}$.

Sequence $\quad N ; K$ combines the exit interface of $N$ with the entry interface of $K$. The entry interface of the resulting box is that of $N$, and the exit interface is that of $K$. For an example see the diagram of $N_{a} ; N_{b}$ in Figure 3.

$$
\begin{gathered}
N ; K=\left(P_{L} \cup P_{R} \cup \mathcal{X}, T_{L} \cup T_{R}\right) \\
\text { where }\left\{\begin{array}{rrr}
T_{L} & =;_{L} \cdot N^{\top} & T_{R}=;_{R} \cdot K^{\top} \\
P_{L} & =;_{L} \cdot N^{\mathrm{ei}} & P_{R}=;_{R} \cdot K^{\mathrm{ix}} \\
\mathcal{X} & =\left\{i_{{ }_{L}} \cdot Z \cup ;_{R} \cdot W\right. & \left.\mathrm{x}_{Z} \in N^{\mathrm{x}} \wedge \mathrm{e}_{W} \in K^{\mathrm{e}}\right\} .
\end{array}\right.
\end{gathered}
$$




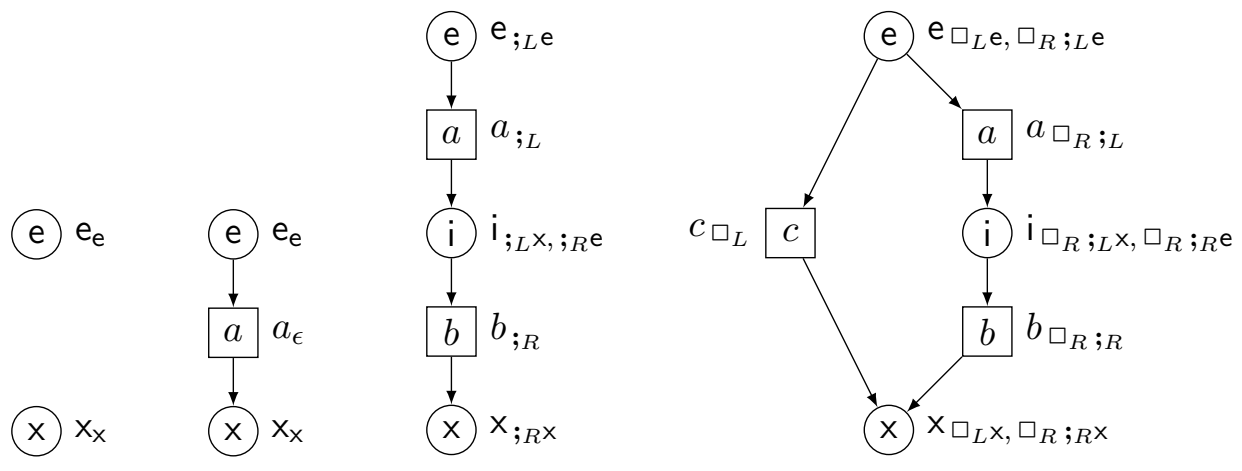

Figure 3. From the left to right: diagrams of $N_{\text {stop }}$ and $N_{a}$ and $N_{a} ; N_{b}$ and $N_{c} \square\left(N_{a} ; N_{b}\right)$. The last two boxes correspond to the box expressions $a ; b$ and $c \square(a ; b)$.

Non-deterministic choice $N \square K$ combines together the entry interfaces of the two boxes creating a new entry interface, as well as their exit interfaces creating a new exit interface. For an example, see the diagram of $N_{c} \square\left(N_{a} ; N_{b}\right)$ in Figure 3 .

$$
\begin{gathered}
N \square K=\left(P_{L} \cup P_{R} \cup \mathcal{X} \cup \mathcal{Y}, T_{L} \cup T_{R}\right) \\
\text { where }\left\{\begin{array}{rrr}
T_{L} & =\square_{L} \cdot N^{\top} & T_{R}=\square_{R} \cdot K^{\top} \\
P_{L} & =\square_{L} \cdot N^{\mathrm{i}} & P_{R}=\square_{R} \cdot K^{\mathrm{i}} \\
\mathcal{X} & =\left\{\mathrm{e}_{\square_{L}} \cdot Z \cup \square_{R} \cdot W\right. & \left.\mid \mathrm{e}_{Z} \in N^{\mathrm{e}} \wedge \mathrm{e}_{W} \in K^{\mathrm{e}}\right\} \\
\mathcal{Y} & =\left\{\mathrm{x}_{\square_{L}} \cdot Z \cup \square_{R} \cdot W\right. & \left.\mid \mathrm{x}_{Z} \in N^{\mathrm{x}} \wedge \mathrm{x}_{W} \in K^{\mathrm{x}}\right\} .
\end{array}\right.
\end{gathered}
$$

Parallelism $\quad N \| K$ simply puts the boxes $N$ and $K$ next to each other. The new entry (resp., exit) interface is the union of the entry (resp., exit) interfaces of the composed boxes.

$$
\begin{gathered}
N \| K=\left(P_{L} \cup P_{R}, T_{L} \cup T_{R}\right) \\
\text { where } \begin{cases}T_{L}={ } \cdot N^{\top} & T_{R}=\|_{R} \cdot K^{\top} \\
P_{L}=\|_{L} \cdot N^{\mathrm{P}} & P_{R}=\|_{R} \cdot K^{\mathrm{P}}\end{cases}
\end{gathered}
$$

Iteration $\llbracket N \circledast K \circledast J \rrbracket$ combines the exit interfaces of $N$ and $K$ with the entry interfaces of $K$ and $J$, respectively. For an example see the diagram of $\llbracket N_{a} \circledast\left(N_{b} \| N_{c}\right) \circledast N_{d} \rrbracket$ in Figure 4(a). The 
(a)
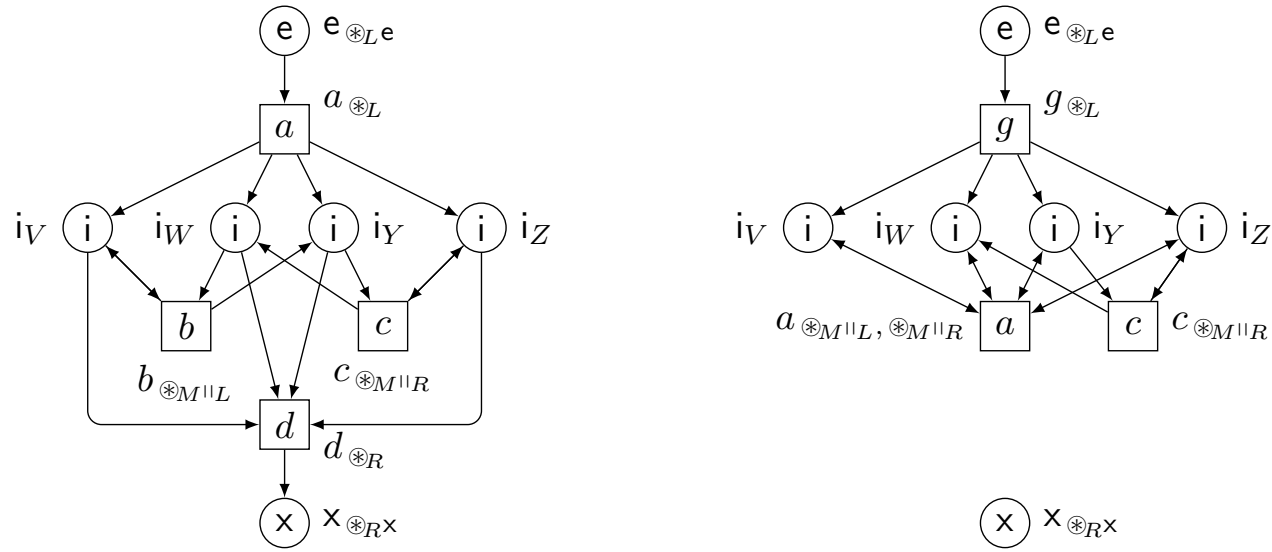

$$
\begin{aligned}
& \mathrm{i}_{V}=\mathrm{i}_{\circledast_{L} \mathrm{x}, \circledast_{M}\left\|_{L} \mathrm{e}, \circledast_{M}\right\|_{L} \mathrm{x}, \circledast_{R} \mathrm{e}} \\
& \mathrm{i}_{Y}=\mathrm{i}_{\circledast_{L} \mathrm{x}, \circledast_{M}\left\|_{R} \mathrm{e}, \circledast_{M}\right\|_{L} \mathrm{x}, \circledast_{R} \mathrm{e}}
\end{aligned}
$$

(x) $x_{\circledast_{R}} \mathrm{x}$

$$
\begin{aligned}
& \mathrm{i}_{W}=\mathrm{i}_{\circledast_{L} \mathrm{x}, \circledast_{M}\left\|_{L} \mathrm{e}, \circledast_{M}\right\|_{R} \mathrm{x}, \circledast_{R} \mathrm{e}} \\
& \mathrm{i}_{Z}=\mathrm{i}_{\circledast_{L} \mathrm{x}, \circledast_{M}\left\|_{R} \mathrm{e}, \circledast_{M}\right\|_{R} \mathrm{x}, \circledast_{R} \mathrm{e}} .
\end{aligned}
$$

Figure 4. Diagrams of two boxes involving parallel composition and iteration: $\llbracket N_{a} \circledast\left(N_{b} \| N_{c}\right) \circledast N_{d} \rrbracket$ (a), and $\left(\llbracket N_{a} \circledast\left(N_{b} \| N_{c}\right) \circledast N_{d} \rrbracket\right) \operatorname{sco}\{a \mapsto g, b c \mapsto a, c \mapsto c\}$ (b). The four internal places are defined below the diagrams.

new entry interface is that of $N$, and the exit interface is that of $J$.

$$
\begin{aligned}
& \llbracket N \circledast K \circledast J \rrbracket=\left(P_{L} \cup P_{M} \cup P_{R} \cup \mathcal{X}, T_{L} \cup T_{M} \cup T_{R}\right) \\
& \text { where }\left\{\begin{array}{rrrr}
T_{L} & =\circledast_{L} \cdot N^{\top} \quad T_{M}=\circledast_{M} \cdot K^{\top} \quad T_{R}=\circledast_{R} \cdot J^{\top} \\
P_{L} & =\circledast_{L} \cdot N^{\mathrm{e}} \quad P_{M}=\circledast_{M} \cdot K^{\mathrm{i}} \quad P_{R}=\circledast_{R} \cdot J^{\mathrm{ix}} \\
\mathcal{X} & =\mathrm{i}_{\circledast_{L}} \cdot Z \cup \circledast_{M} \cdot W \cup \circledast_{M} \cdot V \cup \circledast_{R} \cdot Y \mid \\
& \left.\mathrm{x}_{Z} \in N^{\mathrm{x}} \wedge \mathrm{e}_{W} \in K^{\mathrm{e}} \wedge \mathrm{x}_{V} \in K^{\mathrm{x}} \wedge \mathrm{e}_{Y} \in J^{\mathrm{e}}\right\} .
\end{array}\right.
\end{aligned}
$$

At this point it is possible to formulate a useful result which holds for all boxes which can be constructed using the rules defined so far (clearly, any net constructed in this way is a box).

Proposition 2.1. Let $N$ be any net constructed from boxes corresponding to the constant box expressions as well as the operators for sequence, choice, parallelism, and iteration. Then, the set of steps appearing in the step sequences of $N$ is included in the following set:

$$
N^{\text {psteps }}=\left\{\left\{a_{\pi_{1}}^{1}, \ldots, a_{\pi_{n}}^{n}\right\} \subseteq N^{\top}\left|\forall i<j: \pi_{i}\right| \pi_{j}\right\} .
$$

Moreover, if the net $N_{\text {stop }}$ defined in (2) is not used in the construction, then $N^{\text {psteps }}$ is exactly the set of steps appearing in the step sequences of $N$.

\section{Proof:}

Follows by structural induction from the general results proved, e.g., in [13] for Box Algebra models. 
The last result states that the valid steps of $N$ (i.e., those occurring in the step sequences of $N$ ) do not contain conflicting or causally dependent transitions, i.e., transitions whose syntactic paths $\pi$ and $\pi^{\prime}$ do not satisfy $\pi \mid \pi^{\prime}$. Henceforth we will call $N^{\text {psteps }}$ the set of potential steps of $N$. Moreover, for every synchronisation relation $\rho$, we define:

$$
\rho_{N}=\left\{\left(U, a_{\pi_{1}, \ldots, \pi_{n}}\right) \mid U=\left\{a_{\pi_{1}}^{1}, \ldots, a_{\pi_{n}}^{n}\right\} \in N^{\text {psteps }} \wedge\left(a^{1}, \ldots, a^{n}, a\right) \in \rho\right\} .
$$

Each pair $\left(U, a_{W}\right) \in \rho_{N}$ comprises a set of transitions $U$ which in principle might occur simultaneously in a valid step of $N$ and, under the synchronisation specified by $\rho$ in $N \operatorname{sco} \rho$, would give rise to a compound transition $a_{W}$. For example, if we take the box $N$ in Figure 4(a) with the synchronisation relation $\rho=\{a \mapsto g, b c \mapsto a, c \mapsto c\}$, then we have:

$$
\begin{aligned}
N^{\text {psteps }} & =\left\{\varnothing,\left\{a_{\circledast_{L}}\right\},\left\{b_{\circledast_{M}{ }^{\prime \prime}}\right\},\left\{c_{\circledast_{M} \|_{R}}\right\},\left\{d_{\circledast_{R}}\right\},\left\{b_{\circledast_{M} \|_{L}}, c_{\circledast_{M^{\prime} R}}\right\}\right\} \\
\rho_{N} & =\left\{\left(\left\{a_{\circledast_{L}}\right\}, g_{\circledast_{L}}\right),\left(\left\{c_{\circledast_{M} \|_{R}}\right\}, c_{\circledast_{M} \|_{R}}\right),\left(\left\{b_{\circledast_{M} \|_{L}}, c_{\circledast_{M} \|_{R}}\right\}, a_{\circledast_{M}\left\|_{L}, \circledast_{M}\right\|_{R}}\right)\right\} .
\end{aligned}
$$

Scoping $N \operatorname{sco} \rho$ has the same places as $N$ and, for each potential step of $N$, one creates a new transition representing a synchronisation of two or more actions of $N$, if the potential step is not a singleton. After that, all the transitions of $N$ are removed. Formally,

$$
N \operatorname{sco} \rho=\left(N^{\mathrm{P}}, \mathcal{Z}\right)
$$

where $\mathcal{Z}=\left\{t \mid \exists U:(U, t) \in \rho_{N}\right\}$.

As an example, the diagram of $\llbracket N_{a} \circledast\left(N_{b} \| N_{c}\right) \circledast N_{d} \rrbracket \operatorname{sco}\{a \mapsto g, b c \mapsto a, c \mapsto c\}$ is depicted in Figure 4(b).

As argued in [13], suitable synchronisation relations can capture a wide range of synchronisation schemes of two or more actions (e.g., $b c \mapsto a$ above), as well as action relabelling (e.g., $a \mapsto g$ and $c \mapsto c$ ) and action restriction (as with $d$ ).

\subsection{From expressions to boxes}

We can now define the semantics of box expressions by transforming them compositionally into the corresponding box nets, and then adopting the execution semantics of the latter. Formally, we define a mapping box(.) from expressions to boxes, in the following way:

$$
\begin{aligned}
\operatorname{box}(\operatorname{stop}) & =N_{\text {stop }} \\
\operatorname{box}(a) & =N_{a} \\
\operatorname{box}\left(E ; E^{\prime}\right) & =\operatorname{box}(E) ; \operatorname{box}\left(E^{\prime}\right) \\
\operatorname{box}\left(E \square E^{\prime}\right) & =\operatorname{box}(E) \square \operatorname{box}\left(E^{\prime}\right) \\
\operatorname{box}\left(E \| E^{\prime}\right) & =\operatorname{box}(E) \| \operatorname{box}\left(E^{\prime}\right) \\
\operatorname{box}\left(\llbracket E \circledast E^{\prime} \circledast E^{\prime \prime} \rrbracket\right) & =\llbracket \operatorname{box}(E) \circledast \operatorname{box}\left(E^{\prime}\right) \circledast \operatorname{box}\left(E^{\prime \prime}\right) \rrbracket \\
\operatorname{box}(E \operatorname{sco} \rho) & =\operatorname{box}(E) \operatorname{sco} \rho
\end{aligned}
$$


From now on, by a box we will mean a composite box constructed using (3). According to the BA theory, such boxes enjoy a number of interesting behavioural properties when we consider executions starting from their initial markings as follows (the facts listed below follow from the general results proved in [13] for more Box Algebra models):

- The number of tokens on any place for any reachable marking is bounded; more precisely, the number of tokens on any internal place is never greater than two (i.e., the internal places are 2-bounded), and an entry or exit place never holds more than one token (i.e., the entry and exit places are 1-bounded).

- Each box is clean, which means that when all the exit places contain tokens, there is no token left elsewhere in the net.

- Boxes do not allow auto-concurrency, which means that there is no transition enabled 'twice' for any reachable marking. As a consequence, steps can be represented by sets rather than by multiset of transitions.

The complexity of semantical representations of box expressions provided by the box(.) mapping is in the worst case exponential. To show this, let us consider a synchronised expression $F=E$ sco $\rho$. We define its size $|F|$ as $|E|+|\rho|$, where $|E|$ is the size of $E$ taken to be the total number of occurrences of the stop's and $a$ 's within $E$, and $|\rho|$ to be the total number of action occurrences within $|\rho|$. Moreover, we define the size of a box $N$ as $|N|=\left|N^{\mathrm{P}}\right|+\left|N^{\mathrm{T}}\right|+\left|N^{\mathrm{F}}\right|$. We first observe that $\left|\operatorname{box}(E)^{\mathrm{P}}\right|$, $\left|\operatorname{box}(E)^{\mathrm{T}}\right|$, and $\left|\operatorname{box}(E)^{\mathrm{F}}\right|$ all belong to $O\left(2^{|E|}\right)$. Hence $|\operatorname{box}(E)| \in O\left(2^{|E|}\right)$. As far as $F$ is concerned, we observe that $\left|\operatorname{box}(F)^{\mathrm{P}}\right|=\left|\operatorname{box}(E)^{\mathrm{P}}\right| \in O\left(2^{|E|}\right),\left|\operatorname{box}(F)^{\mathrm{T}}\right| \in O\left(2^{|E|} \cdot|\rho|\right)$, and so $\left|\operatorname{box}(F)^{\mathrm{F}}\right|$ also belongs to $O\left(2^{|E|} \cdot|\rho|\right)$. Hence $|\operatorname{box}(F)| \in O\left(2^{|E|} \cdot|\rho|\right)$. These estimates cannot be much improved which can be shown by taking a sequence of synchronised box expressions $F_{n}=E_{n} \operatorname{sco} \rho_{n}$ where, for every $n \geq 1$ :

$$
E_{n}=(\underbrace{(b \| b) \square \ldots \square(b \| b)}_{n \text { times }}) \|(\underbrace{a\|\ldots\| a}_{n \text { times }}) \text { and } \rho_{n}=\{b \mapsto b\} \cup\{\underbrace{a \ldots a}_{k \text { times }} \mapsto a \mid 1 \leq k \leq n\} .
$$

We first observe that $\left|E_{n}\right|=3 \cdot n,\left|\rho_{n}\right|=2+\frac{1}{2} \cdot n \cdot(n+3)$, and $\left|F_{n}\right|=4 \cdot n+1$. On the other hand:

$$
\begin{array}{rlrlrl}
\left|\operatorname{box}\left(E_{n}\right)^{\mathrm{P}}\right| & =2^{n+1}+2 \cdot n & & \left|\operatorname{box}\left(E_{n}\right)^{\mathrm{P}}\right|=2^{n+1} & +2 \cdot n \\
\left|\operatorname{box}\left(E_{n}\right)^{\mathrm{T}}\right| & =2 \cdot n & +n & \left|\operatorname{box}\left(F_{n}\right)^{\mathrm{T}}\right|=2 \cdot n & +2^{n}-1 \\
\left|\operatorname{box}\left(E_{n}\right)^{\mathrm{F}}\right|=n \cdot 2^{n+1}+2 \cdot n & & \left|\operatorname{box}\left(F_{n}\right)^{\mathrm{F}}\right|=n \cdot 2^{n+1}+\sum_{k=1}^{n} k \cdot\left(\begin{array}{l}
n \\
k
\end{array}\right) .
\end{array}
$$

\subsection{Behavioural properties of composite boxes}

Step sequences of composite boxes exhibit compositional properties [13], i.e., one can (easily) derive the semantics of a composite box from the semantics of the boxes being composed. This is demonstrated by a series of results which follow from the general properties of boxes [13].

For boxes modelling the blocking expression and a single-action expression, the semantics capture is straightforward. 


\section{Proposition 2.2. (basic boxes)}

The following hold, where $a \in \mathcal{A}$ :

$$
\begin{array}{ll}
\operatorname{infsts}\left(N_{\text {stop }}\right)=\varnothing^{\omega} & \operatorname{infsts}\left(N_{a}\right)=\varnothing^{\omega} \cup \varnothing^{*} \cdot\left\{\left\{a_{\epsilon}\right\}\right\} \cdot \varnothing^{\omega} \\
\operatorname{finsts}\left(N_{\text {stop }}\right)=\varnothing \quad & \operatorname{finsts}\left(N_{a}\right)=\varnothing^{*} \cdot\left\{\left\{a_{\epsilon}\right\}\right\} \cdot \varnothing^{*} .
\end{array}
$$

\section{Proof:}

Follows from the general results proved, e.g., in [13].

For choice and parallelism, the semantics of a composite box can easily be expressed in terms of the semantics of the composed boxes. We need, however, a notion of parallel composition of step sequences.

For two step sequences, $\theta$ and $\delta$, of equal length, $\theta \| \delta$ is a step sequence of the same length as $\theta$ and $\delta$, and $(\theta \| \delta)_{(k)}=\theta_{(k)} \cup \delta_{(k)}$, for all $k \leq|\delta|=|\theta|$. Moreover, for two sets of step sequences, $\Theta$ and $\Delta, \Theta \| \Delta=\{\theta \| \delta|\theta \in \Theta \wedge \delta \in \Delta \wedge| \delta|=| \theta \mid\}$.

\section{Proposition 2.3. (choice and parallelism)}

The following hold, where $s t s=$ infsts or $s t s=$ finsts:

$$
\begin{array}{rllll}
\operatorname{sts}(N \square K) & =\square_{L} \cdot \operatorname{sts}(N) & \cup & \square_{R} \cdot \operatorname{sts}(N) \\
\operatorname{sts}(N \| K) & =\|_{L} \cdot \operatorname{sts}(N) & \| & \|_{R} \cdot \operatorname{sts}(N) .
\end{array}
$$

\section{Proof:}

Follows from the general results proved, e.g., in [13].

\section{Proposition 2.4. (sequence)}

The following hold:

$$
\begin{aligned}
& \operatorname{infsts}(N ; K)=;_{L} \cdot \operatorname{finsts}(N) \cdot ;_{R} \cdot \operatorname{infsts}(K) \cup ;_{L} \cdot \operatorname{infsts}(N) \\
& \operatorname{finsts}(N ; K)=;{ }_{L} \cdot \text { finsts }(N) \cdot ;_{R} \cdot \text { finsts }(K) .
\end{aligned}
$$

\section{Proof:}

Follows from the general results proved, e.g., in [13].

\section{Proposition 2.5. (iteration)}

The following hold:

$$
\begin{aligned}
\operatorname{infsts}(\llbracket N \circledast K \circledast J \rrbracket)= & \circledast_{L} \cdot \operatorname{infsts}(N) \cup \\
& \circledast_{L} \cdot \operatorname{finsts}(N) \cdot\left(\circledast_{M} \cdot \operatorname{finsts}(K)\right)^{*} \cdot \circledast_{M} \cdot \operatorname{infsts}(K) \cup \\
& \circledast_{L} \cdot \operatorname{finsts}(N) \cdot\left(\circledast_{M} \cdot \operatorname{finsts}(K)\right)^{*} \cdot \circledast_{R} \cdot \operatorname{infsts}(J) \\
\operatorname{finsts}(\llbracket N \circledast K \circledast J \rrbracket)= & \circledast_{L} \cdot \operatorname{finsts}(N) \cdot\left(\circledast_{M} \cdot \operatorname{finsts}(K)\right)^{*} \cdot \circledast_{R} \cdot \operatorname{finsts}(J) .
\end{aligned}
$$

\section{Proof:}

Follows from the general results proved, e.g., in [13]. 
Finally, we consider a box $N \operatorname{sco} \rho$, where $N$ is constructed from some non-synchronised box expression. In this case, relating step sequences of $N$ sco $\rho$ and $N$ is more involved.

First, we define a relation $\widetilde{\rho}_{N}$ comprising all pairs $\left(U,\left\{t_{1}, \ldots, t_{k}\right\}\right)(k \geq 0)$, where $U \in N^{\text {psteps }}$ and $\left\{t_{1}, \ldots, t_{k}\right\} \subseteq \operatorname{box}(N \operatorname{sco} \rho)^{\top}$ are such that there is a partition $U_{1}, \ldots, U_{k}$ of $U$ satisfying $\left(U_{j}, t_{j}\right) \in \rho_{N}$, for each $j \leq k$. Moreover, for two equal length sequences of sets of transitions, $\tau$ and $\theta$, we denote $(\tau, \theta) \in \widetilde{\rho}_{N}$ if $\left(\tau_{(j)}, \theta_{(j)}\right) \in \widetilde{\rho}_{N}$, for all $j$.

Proposition 2.6. (scoping)

$\operatorname{sts}(N \operatorname{sco} \rho)=\left\{\theta \mid \exists \tau \in \operatorname{sts}(N):(\tau, \theta) \in \widetilde{\rho}_{N}\right\}$, where $s t s=$ infsts or sts $=$ finsts.

\section{Proof:}

Follows from the general results proved, e.g., in [13].

\subsection{Streamlined box expressions}

The translation from BA to ITL, that will be described in Section 4, is particularly simple for the class of streamlined synchronised expressions.

We first observe that each transition $a_{W}$ in the box associated with a non-synchronized box expression is such that $W$ is a singleton. Thus, $a_{W}$ is represented as $a_{\pi}$ for a syntax path $\pi$.

We call a box expression $E \operatorname{sco} \rho$ streamlined if for each transition $a_{\pi} \in \operatorname{box}(E)^{\top}$ there is exactly one transition $b_{W} \in \operatorname{box}(E \operatorname{sco} \rho)^{\top}$ such that $\pi \in W$. That is, any pre-synchronisation action in a streamlined box expression contributes to exactly one action after applying the synchronisation. ${ }^{3}$

We will now demonstrate that each synchronised box expression $F=E$ sco $\rho$ can be transformed into a semantically equivalent ${ }^{4}$ streamlined expression $\operatorname{stl}(F)=F^{\prime}=E^{\prime} \operatorname{sco} \rho^{\prime}$. First, for every $a_{\pi} \in \operatorname{box}(E)^{\top}$, let

$$
\operatorname{trans}\left(a_{\pi}\right)=\left\{b_{W} \in \operatorname{box}(F)^{\top} \mid \pi \in W\right\}=\left\{t \mid \exists(U, t) \in \rho_{\text {box }(E)}: a_{\pi} \in U\right\} .
$$

In other words, trans $\left(a_{\pi}\right)$ comprises all post-synchronisation transitions in which $a_{\pi}$ has been involved. For example, if we take $\llbracket a \circledast(b \| c) \circledast d \rrbracket \operatorname{sco}\{a \mapsto g, b c \mapsto a, c \mapsto c\}$ with the corresponding box depicted in Figure 4(b), we have:

$$
\begin{aligned}
\operatorname{trans}\left(a_{\circledast_{L}}\right) & =\left\{g_{\circledast_{L}}\right\} & \operatorname{trans}\left(b_{\circledast_{M}{ }^{\prime} L}\right) & =\left\{a_{\circledast_{M}{ }_{L}}, \circledast_{M}{ }_{M}\right\} \\
\operatorname{trans}\left(d_{\circledast_{R}}\right) & =\varnothing & \operatorname{trans}\left(c_{\circledast_{M} \|_{R}}\right) & =\left\{a_{\circledast_{M} \|_{L}}, \circledast_{M}{ }_{M}, c_{\circledast_{M} \|_{R}}\right\}
\end{aligned}
$$

Then, a suitable $E^{\prime}$ is obtained by replacing each occurrence of an action $a \in \mathcal{A}$ in $E$ corresponding to transition $a_{\pi}$ in box $(E)^{5}$ by:

- stop if we have $\operatorname{trans}\left(a_{\pi}\right)=\varnothing$,

- $b_{W}$ if we have $\operatorname{trans}\left(a_{\pi}\right)=\left\{b_{W}\right\}$, and

- $b_{W_{1}}^{1} \square\left(\ldots \square\left(b_{W_{m-1}}^{m-1} \square b_{W_{m}}^{m}\right) \ldots\right)$ if we have trans $\left(a_{\pi}\right)=\left\{b_{W_{1}}^{1}, \ldots, b_{W_{m}}^{m}\right\}$ and $m \geq 2^{6}$.

\footnotetext{
${ }^{3}$ As a result, each pre-synchronisation action can be represented by a single variable in the corresponding ITL formula.

${ }^{4}$ In the sense of generating isomorphic box net with 'isomorphic' step sequences, see Proposition 2.7.

${ }^{5}$ Such an occurrence of $a$ is identified by the path in the syntax tree of the non-synchronised expression $E$ which corresponds to $\pi$.

${ }^{6} \mathrm{We}$ assume a fixed ordering on the transitions of $\operatorname{box}(F)$ so that the enumeration of $\operatorname{trans}\left(a_{\pi}\right)$ is unique.
} 
Furthermore, we modify the scoping part reflecting changes affecting the set of actions:

$$
\rho^{\prime}=\{\underbrace{b_{W} \ldots b_{W}}_{|W| \text { times }} \mapsto b_{W} \mid b_{W} \in \operatorname{box}(F)^{\top}\}
$$

The $b_{W} \ldots b_{W} \mapsto b_{W}$ above reflects the fact that $b_{W}$ is constructed as a synchronisation of $|W|$ actions in box $(E)$, each such action being now replaced by a copy of $b_{W}$ in $E^{\prime}$.

Continuing the last example, we obtain $F^{\prime}=E^{\prime}$ sco $\rho^{\prime}$, with

$$
E^{\prime}=(\llbracket \gamma \circledast(\alpha \|(\alpha \square \zeta)) \circledast \text { stop } \rrbracket) \quad \text { and } \quad \rho^{\prime}=\{\gamma \mapsto \gamma, \alpha \alpha \mapsto \alpha, \zeta \mapsto \zeta\} \text {, }
$$

where $\gamma=g_{\circledast_{L}}, \alpha=a_{\circledast_{M} \|_{L}}, \circledast_{M} \|_{R}$, and $\zeta=c_{\circledast_{M} \|_{R}}$.

Each transition of box $\left(F^{\prime}\right)$ is of the form $\left(b_{W}\right)_{Y}$, where $b_{W} \in \operatorname{box}(E \operatorname{sco} \rho)^{\top}{ }^{7}$, and we then define a bijection $\lambda: \operatorname{box}\left(F^{\prime}\right)^{\top} \rightarrow \operatorname{box}(F)^{\top}$ by setting $\lambda\left(\left(b_{W}\right)_{Y}\right)=b_{W}$. Such a $\lambda$ can be applied in the standard way to the subsets of box $\left(F^{\prime}\right)^{\top}$ and their sequences.

\section{Proposition 2.7. (streamlined expression)}

The nets box $(F)$ and box $(\operatorname{stl}(F))$ are isomorphic after replacing each transition label $b_{W}$ in box $(\operatorname{stl}(F))$ by $b$. Moreover, sts $(\operatorname{box}(F))=\lambda(\operatorname{sts}(\operatorname{box}(\operatorname{stl}(F))))$, where $s t s=$ infsts or $s t s=$ finsts.

\section{Proof:}

We observe that the sets of places in $\operatorname{box}(F)$ and box $(\operatorname{stl}(F))$ are in a one-to-one correspondence since the subexpressions of the form stop or $b_{W}$ or $b_{W_{1}}^{1} \square\left(\ldots \square\left(b_{W_{m-1}}^{m-1} \square b_{W_{m}}^{m}\right) \ldots\right)$ produce boxes with exactly one entry place, one exit place, and no internal places.

The first part of the result follows from the fact that the result of synchronising $|W|$ transitions in $\operatorname{box}(E)$ to yield a transition $b_{W}$ is the same w.r.t. connections with the (corresponding) places as the result of synchronising $|W|$ transitions labelled $b_{W}$ in box $\left(E^{\prime}\right)$ yielding a transition $\left(b_{W}\right)_{Y}$.

The second part follows from the first part and the fact that $\lambda$ is a part of isomorphism between $\operatorname{box}(F)$ and box $(\operatorname{stl}(F))$.

Streamlined expressions will prove their usefulness in the translation of box expressions to behaviourally equivalent ITL formulas. Intuitively, they allow one to separate the roles that a single variable can play in different synchronisation contexts.

In terms of complexity, the transformation of $F=E \operatorname{sco} \rho$ into a streamlined $\operatorname{stl}(F)=E^{\prime} \operatorname{sco} \rho^{\prime}$ will usually result in a larger expression. This increase is a result of inserting sub-expressions of the form $b_{W_{1}}^{1} \square\left(\ldots \square\left(b_{W_{m-1}}^{m-1} \square b_{W_{m}}^{m}\right) \ldots\right)$ and modifying the scoping relation. It is straightforward to check that $\left|E^{\prime}\right| \in O\left(|E|+\left|\operatorname{box}(E)^{\mathrm{F}}\right|\right)$ and $\left|\rho^{\prime}\right|=\left|\operatorname{box}(E)^{\mathrm{F}}\right|$. Hence $|\operatorname{stl}(F)| \in O\left(|E|+\left|\operatorname{box}(E)^{\mathrm{F}}\right|\right)$, and so $|\operatorname{stl}(F)| \in O(|E|+\mid \operatorname{box}(E))$. Since it can be argued that, for the purpose of behavioural analyses within the domain of Petri nets, the problem size is $|F|+|\operatorname{box}(F)|$, the transformation to streamlined expressions is efficient.

It is also important to stress that it is not necessary to derive box $(F)$ in order to derive $\operatorname{stl}(F)$.

\footnotetext{
${ }^{7}$ In general, $W \neq Y$ since the syntax tree of $E$ is not isomorphic to the syntax tree of $E^{\prime}$ due to the introduction of
} sub-expressions of the form $b_{W_{1}}^{1} \square\left(\ldots \square\left(b_{W_{m-1}}^{m-1} \square b_{W_{m}}^{m}\right) \ldots\right)$. 


\section{Interval Temporal Logic}

We now provide the syntax and semantics of a small fragment of ITL, including only those constructs (basic and derived) which are used in the translation of box expressions. In particular, we assume that Var is a countable set of Boolean variables, all such variables being the transitions of boxes created using the box mapping.

The formulas of the fragment of the ITL logic we need are defined by:

$$
\varphi::=\operatorname{true}|\operatorname{flip}(v)| \operatorname{skipstb}(v)|\varphi \wedge \varphi| \varphi \vee \varphi|\varphi ; \varphi| \varphi^{*} \mid \inf
$$

where $v \in \operatorname{Var}$. Intuitively, flip $(v)$ inverts the value of a Boolean variable $v$ over a unit interval, skipstbl $(v)$ keeps unchanged the value of $v$ over a unit interval, "; " is a sequential composition operator (called chop), “*” is an iterative version of chop (called chop-star), and inf indicates an infinite interval. The set of variables occurring in a formula $\varphi$ is denoted by $\operatorname{var}(\varphi)$. and its size $|\varphi|$ is the total number of occurrences of the $v$ 's, stop's, and inf's within $\varphi$.

Remark 3.1. The logic syntax introduced above has been tailored to smooth the translation from box expression to logical formulas. However, all the non-standard constructs used (i.e., true, flip, skipstbl and inf) can be expressed in the standard ITL logic, in the following way:

$$
\begin{aligned}
\text { true } & =v \vee \neg v & \operatorname{flip}(v) & =(\bigcirc \neg \bigcirc \text { true }) \wedge(v=\bigcirc \neg v) \\
\text { inf } & =\text { true; } \neg \text { true } & \text { skipstbl }(v) & =(\bigcirc \neg \bigcirc \text { true }) \wedge(v=\bigcirc v)
\end{aligned}
$$

where $\bigcirc$ is the temporal operator 'next'. Hence it is possible to formulate and analyse behavioural properties of any translated formula using proof techniques and tools of ITL (e.g., [11] gives a complete axiomatisation of both finite and infinite time).

A state $s$ is a mapping which assigns values to the Boolean variables Var, and an interval $\sigma$ is a non-empty sequence of states. The meaning of formulas is given by the satisfaction relation $=$ involving intervals and formulas, defined as follows (below $s, s^{\prime}$ are states, and $\sigma, \sigma^{\prime}, \sigma^{\prime \prime}$ are intervals):

- $\sigma=$ true,

- $\sigma=\mathrm{flip}(v)$ if $\sigma=s \cdot s^{\prime}$ and $s(v) \neq s^{\prime}(v)$,

- $\sigma=\operatorname{skipstbl}(v)$ if $\sigma=s \cdot s^{\prime}$ and $s(v)=s^{\prime}(v)$,

- $\sigma \models \varphi \vee \varphi^{\prime}$ if $\sigma \models \varphi$ or $\sigma \models \varphi^{\prime}$,

- $\sigma=\varphi \wedge \varphi^{\prime}$ if $\sigma \models \varphi$ and $\sigma \models \varphi^{\prime}$,

- $\sigma=\varphi ; \varphi^{\prime}$ if $\sigma$ is infinite and $\sigma=\varphi$, or $\sigma$ can be decomposed as $\sigma=\sigma^{\prime} \cdot s \cdot \sigma^{\prime \prime}$ so that $\sigma^{\prime} \cdot s \models \varphi$ and $s \cdot \sigma^{\prime \prime} \models \varphi^{\prime}$,

- $\sigma \models \varphi^{*}$ if $|\sigma|=1$, or $\sigma$ can be decomposed as $\sigma=\sigma^{\prime} \cdot s \cdot \sigma^{\prime \prime}$ so that $\sigma^{\prime} \cdot s \mid=\varphi$ and $s \cdot \sigma^{\prime \prime} \mid=\varphi^{*}$, and 
- $\sigma \models \inf$ if $\sigma$ is infinite.

To capture the relationship between the semantics of a box expression and a corresponding formula, with each ITL formula $\varphi$ and interval $\sigma$ satisfying $\sigma \models \varphi$, we associate a sequence of sets $\mu_{\sigma}=\Gamma_{1} \Gamma_{2} \ldots$, where, for each $j<|\sigma|, \Gamma_{j}$ is given by:

$$
\Gamma_{j}=\operatorname{var}(\varphi) \cap\left\{v \in \operatorname{var}(\varphi) \mid \sigma_{(j)}(v) \neq \sigma_{(j+1)}(v)\right\} .
$$

Since each $\Gamma_{j}$ records all the variables which flipped their values at the point of entering the state $\sigma_{(j+1)}$, the sequence $\mu_{\sigma}$ provides a direct interpretation of $\sigma$ in terms of sequences of steps of transitions of box nets. Then, for any ITL formula $\varphi$, we define:

$$
\operatorname{infsts}(\varphi)=\left\{\mu_{\sigma}|\sigma \models \varphi \wedge| \sigma \mid=\omega\right\} \quad \text { and } \quad \text { finsts }(\varphi)=\left\{\mu_{\sigma}|\sigma|=\varphi \wedge|\sigma|<\omega\right\} .
$$

Below we present a number of semantrical properties of ITL formulas considered in this paper.

Proposition 3.2. Let $\varphi$ and $\varphi^{\prime}$ be two formulas with disjoint sets of variables, i.e., $\operatorname{var}(\varphi) \cap \operatorname{var}\left(\varphi^{\prime}\right)=$ $\varnothing$. Then, the following hold, where $\psi=\operatorname{skipstbl}(\operatorname{var}(\varphi))^{*}, \psi^{\prime}=\operatorname{skipstbl}\left(\operatorname{var}\left(\varphi^{\prime}\right)\right)^{*}$, and sts $=$ infsts or $s t s=$ finsts:

$$
\begin{aligned}
\operatorname{sts}\left(\left(\varphi \wedge \psi^{\prime}\right) \vee\left(\varphi^{\prime} \wedge \psi\right)\right) & =\operatorname{sts}(\varphi) \cup \operatorname{sts}\left(\varphi^{\prime}\right) \\
\operatorname{sts}\left(\varphi \wedge \varphi^{\prime}\right) & =\operatorname{sts}(\varphi) \| \operatorname{sts}\left(\varphi^{\prime}\right) \\
\operatorname{infsts}\left(\left(\varphi \wedge \psi^{\prime}\right) ;\left(\varphi^{\prime} \wedge \psi\right)\right) & =\operatorname{infsts}(\varphi) \cup \operatorname{finsts}(\varphi) \cdot \operatorname{infsts}\left(\varphi^{\prime}\right) \\
\operatorname{finsts}\left(\left(\varphi \wedge \psi^{\prime}\right) ;\left(\varphi^{\prime} \wedge \psi\right)\right) & =\operatorname{finsts}(\varphi) \cdot \operatorname{finsts}\left(\varphi^{\prime}\right) .
\end{aligned}
$$

\section{Proof:}

Follows directly from the basic properties of logic operators.

Proposition 3.3. Let $\varphi_{i}$, for $i=1,2,3$, be formulas with mutually disjoint sets of variables. Then, the following hold, where $\psi_{i, j}=\operatorname{skipstbl}\left(\operatorname{var}\left(\varphi_{i}\right) \cup \operatorname{var}\left(\varphi_{j}\right)\right)^{*}$, for $i, j \in\{1,2,3\}$ :

$$
\begin{array}{ll}
\operatorname{finsts}\left(\left(\varphi_{1} \wedge \psi_{2,3}\right) ;\left(\left(\varphi_{2} \wedge \psi_{1,3}\right)^{*} ;\left(\varphi_{3} \wedge \psi_{1,2}\right)\right)\right)= & \operatorname{finsts}\left(\varphi_{1}\right) \cdot \operatorname{finsts}\left(\varphi_{2}\right)^{*} \cdot \operatorname{finsts}\left(\varphi_{3}\right) \\
\operatorname{infsts}\left(\left(\varphi_{1} \wedge \psi_{2,3}\right) ;\left(\left(\varphi_{2} \wedge \psi_{1,3}\right)^{*} ;\left(\varphi_{3} \wedge \psi_{1,2}\right)\right)\right)= & \operatorname{infsts}\left(\varphi_{1}\right) \cup \\
& \operatorname{finsts}\left(\varphi_{1}\right) \cdot \operatorname{finsts}\left(\varphi_{2}\right)^{*} \cdot\left(\operatorname{infsts}\left(\varphi_{2}\right) \cup \operatorname{infsts}\left(\varphi_{3}\right)\right) .
\end{array}
$$

\section{Proof:}

We obtain the following, after noting that $\operatorname{var}\left(\varphi_{2}^{*}\right)=\operatorname{var}\left(\varphi_{2}\right)$ and twice using Proposition 3.2:

$$
\begin{aligned}
\operatorname{finsts} & \left(\left(\varphi_{1} \wedge \psi_{2,3}\right) ;\left(\left(\varphi_{2} \wedge \psi_{1,3}\right)^{*} ;\left(\varphi_{3} \wedge \psi_{1,2}\right)\right)\right. \\
& =\operatorname{finsts}\left(\left(\varphi_{1} \wedge \psi_{2,3}\right) ;\left(\left(\left(\varphi_{2} \wedge \psi_{3,3}\right)^{*} ;\left(\varphi_{3} \wedge \psi_{2,2}\right)\right) \wedge \psi_{1,1}\right)\right. \\
& =\operatorname{finsts}\left(\varphi_{1}\right) \cdot \text { finsts }\left(\left(\varphi_{2} \wedge \psi_{3,3}\right)^{*} ;\left(\varphi_{3} \wedge \psi_{2,2}\right)\right) \\
& =\operatorname{finsts}\left(\varphi_{1}\right) \cdot \operatorname{finsts}\left(\left(\varphi_{2}^{*} \wedge \psi_{3,3}\right) ;\left(\varphi_{3} \wedge \operatorname{skipstbl}\left(\operatorname{var}\left(\varphi_{2}^{*}\right)\right)^{*}\right)\right) \\
& =\operatorname{finsts}\left(\varphi_{1}\right) \cdot \text { finsts }\left(\varphi_{2}^{*}\right) \cdot \operatorname{finsts}\left(\varphi_{3}\right) \\
& =\operatorname{finsts}\left(\varphi_{1}\right) \cdot \operatorname{finsts}\left(\varphi_{2}\right)^{*} \cdot \operatorname{finsts}\left(\varphi_{3}\right) .
\end{aligned}
$$

The second part of the proof is similar. 
Proposition 3.4. Let $\varphi^{\prime}$ be a formula obtained from an ITL formula $\varphi$ by a consistent renaming of Boolean variables given by a bijection $\lambda$. Then $s t s\left(\varphi^{\prime}\right)=\lambda(s t s(\varphi))$, for $s t s=$ infsts or $s t s=$ finsts.

\section{Proof:}

Follows from the insensitivity of $\mid=$ to the identities of logic variables.

For a formula $\varphi$ and $\pi \in \Pi$, we will denote by $\pi \cdot \varphi$ the formula obtained from $\varphi$ by replacing each variable $a_{\pi^{\prime}}$ with $a_{\pi \cdot \pi^{\prime}}$.

Proposition 3.5. If $\pi \in \Pi$ then $s t s(\pi \cdot \varphi)=\pi \cdot s t s(\varphi)$, where $s t s=$ infsts or $s t s=$ finsts.

\section{Proof:}

This follows from Proposition 3.4 and the fact that the transformation given by $\pi \cdot \varphi$ is a consistent renaming of variables.

\subsection{Derived formulas}

and we will use the following derived formulas, for finite sets of variables $V, V^{\prime} \subseteq \operatorname{Var}$ :

$$
\begin{aligned}
\text { skipstbl}(V) & = \begin{cases}\bigwedge_{v \in V} \operatorname{skipstbl}(v) & \text { if } V \neq \varnothing \\
\text { true } & \text { otherwise }\end{cases} \\
\operatorname{infstbl}(V) & =\inf \wedge \operatorname{skipstbl}(V)^{*} \\
\operatorname{flip}(V) & = \begin{cases}\bigvee_{v \in V} \operatorname{flip}(v) \wedge \operatorname{skipstbl}(V \backslash\{v\}) & \text { if } V \neq \varnothing \\
\inf & \text { otherwise }\end{cases} \\
\mathrm{fs}\left(V \mid V^{\prime}\right) & =\text { skipstbl }\left(V \cup V^{\prime}\right)^{*} ;\left(\operatorname{flip}(V) \wedge \operatorname{skipstbl}\left(V^{\prime}\right)\right) ; \text { skipstbl }\left(V \cup V^{\prime}\right)^{*} .
\end{aligned}
$$

Intuitively, skipstbl $(V)$ and infstbl $(V)$ keep unchanged the values of the variables in $V$ respectively over a unit and infinite interval, flip $(V)$ inverts the value of exactly one of the Boolean variables in $V$ over a unit interval, and $\mathrm{fs}\left(V \mid V^{\prime}\right)$ keeps unchanged the values of the variables in $V$ and $V^{\prime}$ over an interval except that it inverts the value of exactly one variable in $V$ over a unit sub-interval. We can drop the set parenthesis when writing down the above formulas.

Using the derived syntax, we call an ITL logical formula an infstbl/fs-formula if it constructed using infstbl $(V)$ 's, fs $\left(V \mid V^{\prime}\right)$ 's and the four logical operators of the syntax. The translation we will introduce in the next section will translate box expressions into infstbl/fs-formulas.

It is easily checked that the following hold for any finite sets of variables $V, V^{\prime}, V^{\prime \prime} \subseteq \operatorname{Var}$ satisfying $V \cap V^{\prime \prime}=\varnothing$, where $\equiv$ denotes the standard equivalence of formulas w.r.t. the satisfaction relation $\models$ :

$$
\begin{array}{ll}
\mathrm{fs}(\varnothing \mid V) \equiv \operatorname{infstbl}(V) & \operatorname{infstbl}(V) \wedge \operatorname{skipstbl}\left(V^{\prime}\right)^{*} \equiv \operatorname{infstbl}\left(V \cup V^{\prime}\right) \\
\mathrm{fs}(V \mid V) \equiv \operatorname{infstbl}(V) & \mathrm{fs}\left(V \mid V^{\prime}\right) \wedge \operatorname{skipstbl}\left(V^{\prime \prime}\right)^{*} \equiv \mathrm{fs}\left(V \mid V^{\prime} \cup V^{\prime \prime}\right) .
\end{array}
$$


Moreover, skipstbl $(V)$ distributes over the logical operators as we have the following, for all formulas $\varphi, \varphi^{\prime}$ and finite sets of variables $V$ :

$$
\begin{aligned}
& \left(\varphi \wedge \varphi^{\prime}\right) \wedge \operatorname{skipstbl}(V) \equiv\left(\varphi ; \varphi^{\prime}\right) \wedge \operatorname{skipstbl}(V) \\
& \left(\varphi \wedge \varphi^{\prime}\right) \vee \operatorname{skipstbl}(V) \equiv(\varphi \wedge \operatorname{skipstbl}(V)) \vee\left(\varphi^{\prime} \wedge \operatorname{skipstbl}(V)\right) \\
& \left(\varphi \wedge \varphi^{\prime}\right) ; \operatorname{skipstbl}(V) \equiv(\varphi \wedge \operatorname{skipstbl}(V)) ;\left(\varphi^{\prime} \wedge \operatorname{skipstbl}(V)\right)
\end{aligned}
$$

For every infstbl/fs-formula $\varphi$ and a finite set of variables $V$, we will denote by $V \rightarrow \varphi$ the formula obtained by replacing each sub-formula infstbl $\left(V^{\prime}\right)$ by infstbl $\left(V \cup V^{\prime}\right)$, and each sub-formula fs $\left(V^{\prime} \mid V^{\prime \prime}\right)$ by fs $\left(V^{\prime} \mid V \cup V^{\prime \prime}\right)$. By (5) and (6), we immediately obtain that:

$$
V \triangleright \varphi \equiv \varphi \wedge \operatorname{skipstbl}(V)^{*} .
$$

\section{From box expressions to logical formulas}

To make the presentation more accessible, we will first show how to translate non-synchronised box expressions. After that, we will extend the translation to the streamlined synchronised expressions, and, finally, we will deal with the case of general synchronised expressions.

\subsection{Translating non-synchronised expressions}

The translation for non-synchronised expressions yields infstbl/fs-formulas, and is defined compositionally in the following way:

$$
\begin{aligned}
& \operatorname{itl}(\text { stop })=\operatorname{infstbl}(\varnothing) \\
& \operatorname{itl}(a)=\mathrm{fs}\left(a_{\epsilon} \mid \varnothing\right) \\
& \operatorname{itl}(E ; F)=\left(\left(;_{R} \cdot \operatorname{var}(\operatorname{itl}(F))\right)>\left(;_{L} \cdot \operatorname{itt}(E)\right)\right) ;\left(\left(;_{L} \cdot \operatorname{var}(\operatorname{itl}(E))\right)>\left(;_{R} \cdot \operatorname{itl}(F)\right)\right) \\
& \operatorname{itl}(E \square F)=\left(\left(\square_{R} \cdot \operatorname{var}(\mathrm{itl}(F))\right) \vee\left(\square_{L} \cdot \mathrm{itl}(E)\right)\right) \vee\left(\left(\square_{L} \cdot \operatorname{var}(\mathrm{itl}(E))\right) \vee\left(\square_{R} \cdot \mathrm{itl}(F)\right)\right) \\
& \operatorname{itl}(E \| F)=\left\|_{L} \cdot \operatorname{itl}(E) \wedge\right\|_{R} \cdot \operatorname{itl}(F) \\
& \operatorname{itl}(\llbracket E \circledast F \circledast G \rrbracket)=\left(\left(\left(\circledast_{M} \cdot \operatorname{var}(\operatorname{itl}(F))\right) \cup\left(\circledast_{R} \cdot \operatorname{var}(\mathrm{itl}(G))\right)\right)>\left(\circledast_{L} \cdot \operatorname{itl}(E)\right)\right) \text {; } \\
& \left(\left(\left(\circledast_{L} \cdot \operatorname{var}(\mathrm{itl}(E))\right) \cup\left(\circledast_{R} \cdot \operatorname{var}(\mathrm{itl}(G))\right)\right)>\left(\circledast_{M} \cdot \mathrm{itl}(F)\right)\right)^{*} ; \\
& \left(\left(\left(\circledast_{L} \cdot \operatorname{var}(\mathrm{itl}(E))\right) \cup\left(\circledast_{M} \cdot \operatorname{var}(\mathrm{itl}(F))\right)\right)>\left(\circledast_{R} \cdot \mathrm{itl}(G)\right)\right) \text {. }
\end{aligned}
$$

As an example, for the expressions generating the boxes in Figure 3, we obtain:

$$
\begin{aligned}
\operatorname{itl}(a ; b) & =\mathrm{fs}\left(a_{;_{L}} \mid b_{;_{R}}\right) ; \mathrm{fs}\left(b_{;_{R}} \mid a_{;_{L}}\right) \\
\operatorname{itl}(c \square(a ; b)) & =\left(\mathrm{fs}\left(c_{\square_{L}} \mid a_{\square_{R} ; L}, b_{\square_{R} ; R}\right)\right) \vee\left(\mathrm{fs}\left(a_{\square_{R} ;_{L}} \mid b_{\square_{R} ; R}, c_{\square_{L}}\right) ; \mathrm{fs}\left(b_{\square_{R} ; R} \mid a_{\square_{R} ; L}, c_{\square_{L}}\right)\right) .
\end{aligned}
$$

As far as the complexity of the translation is concerned, $|i t|(E) \mid \in O\left(|E|^{2}\right)$. This compares very favourably with the size of the original semantical representation of $E$ since $|\operatorname{box}(E)| \in O\left(2^{|E|}\right)$.

Crucially, the step semantics of a non-synchronised box expression and the corresponding ITL formula coincide. 


\section{Theorem 4.1. (non-synchronised expression)}

If $H$ is a non-synchronised box expression then $s t s(\operatorname{itl}(H))=s t s(\operatorname{box}(H))$, where $s t s=$ infsts or $s t s=$ finsts.

\section{Proof:}

Let $\psi=\operatorname{itl}(H)$ and $N=\operatorname{box}(H)$. The proof proceeds by induction on the structure of $H$.

Case 1: $H=$ stop. Then $\psi=\inf \equiv \operatorname{infstbl}(\varnothing)$ and $N=N_{\text {stop. }}$ Hence we have:

$$
\operatorname{infsts}(\psi)=\varnothing^{\omega}=\operatorname{infsts}(N) \quad \text { and } \operatorname{finsts}(\psi)=\varnothing=\operatorname{finsts}(N) .
$$

Case 2: $H=a$. Then, $\psi=\mathrm{fs}\left(a_{\epsilon} \mid \varnothing\right)=\operatorname{skipstbl}\left(a_{\epsilon}\right)^{*} ;$ flip $\left(a_{\epsilon}\right) ; \operatorname{skipstbl}\left(a_{\epsilon}\right)^{*}$ and $N=N_{a}$. Hence we have:

$$
\begin{gathered}
\operatorname{infsts}(\psi)=\varnothing^{\omega} \cup \varnothing^{*} \cdot\left\{\left\{a_{\epsilon}\right\}\right\} \cdot \varnothing^{\omega}=\operatorname{infsts}(N) \\
\text { finsts }(\psi)=\varnothing^{*} \cdot\left\{\left\{a_{\epsilon}\right\}\right\} \cdot \varnothing^{*}=\text { finsts }(N) .
\end{gathered}
$$

Case 3: $H=E \square F$. Then, by the equivalence (7), we have:

$$
\psi \equiv \square_{L} \cdot \operatorname{itl}(E) \wedge \operatorname{skipstbl}\left(\square_{R} \cdot \operatorname{var}(\operatorname{itl}(F))\right)^{*} \vee \square_{R} \cdot \operatorname{itl}(F) \wedge \operatorname{skipstbl}\left(\square_{L} \cdot \operatorname{var}(\operatorname{itl}(E))\right)^{*} .
$$

Hence, by $\operatorname{var}(\psi)=\square_{L} \cdot \operatorname{var}(\operatorname{itl}(E)) \cup \square_{R} \cdot \operatorname{var}(\operatorname{itl}(F))$ as well as (applied in this order) Proposition 3.2, Proposition 3.5, the induction hypothesis, and Proposition 2.3, we obtain:

$$
\begin{aligned}
& \operatorname{sts}(\psi)=\operatorname{sts}\left(\square_{L} \cdot \mathrm{itl}(E)\right) \cup \operatorname{sts}\left(\square_{R} \cdot \mathrm{itl}(F)\right) \\
& =\square_{L} \cdot \operatorname{sts}(\operatorname{itl}(E)) \quad \cup \quad \square_{R} \cdot \operatorname{sts}(\operatorname{itl}(F)) \\
& =\square_{L} \cdot s t s(\operatorname{box}(E)) \cup \square_{R} \cdot s t s(\operatorname{box}(F))=s t s(H) \text {. }
\end{aligned}
$$

Case 4: $H=E \| F$. Then, by Proposition 3.2, Proposition 3.5, the induction hypothesis, and Proposition 2.3, we have:

$$
\begin{aligned}
& s t s(\psi)=\operatorname{sts}\left(\|_{L} \cdot \mathrm{itl}(E)\right) \quad \| \quad s t s\left(\|_{L} \cdot \mathrm{it}(F)\right) \\
& ={ }^{\prime} L \cdot \operatorname{sts}(\operatorname{itl}(E)) \quad \| \quad{ }_{L} \cdot \operatorname{sts}(\operatorname{itl}(F)) \\
& =\left\|_{L} \cdot s t s(\operatorname{box}(E)) \quad\right\| \quad \|_{L} \cdot s t s(\operatorname{box}(F))=s t s(H) \text {. }
\end{aligned}
$$

Case 5: $H=E ; F$. Then, by the equivalence (7), we have:

$$
\psi=\quad{ }_{L} \cdot \operatorname{itl}(E) \wedge \operatorname{skipstbl}\left(;_{R} \cdot \operatorname{var}(\operatorname{itl}(F))\right)^{*} \quad ; \quad ;_{R} \cdot \operatorname{itl}(F) \wedge \operatorname{skipstbl}\left(;_{L} \cdot \operatorname{var}(\operatorname{itl}(E))\right)^{*} .
$$

Hence we obtain, by $\operatorname{var}(\psi)=;_{L} \cdot \operatorname{var}(\operatorname{itl}(E)) \cup ;_{R} \cdot \operatorname{var}(\operatorname{itl}(F))$ as well as (applied in this order) Proposition 3.2, Proposition 3.5, the induction hypothesis, and Proposition 2.4:

$$
\begin{aligned}
\operatorname{finsts}(\psi) & =\operatorname{finsts}\left(;_{L} \cdot \operatorname{itl}(E)\right) \cdot \operatorname{finsts}\left(;_{R} \cdot \operatorname{itl}(F)\right) \\
& =;_{L} \cdot \operatorname{finsts}(\operatorname{itl}(E)) \cdot ;_{R} \cdot \operatorname{finsts}(\operatorname{itl}(F)) \\
& =;_{L} \cdot \operatorname{finsts}(\operatorname{box}(E)) \cdot ;_{R} \cdot \operatorname{finsts}(\operatorname{box}(F))=\operatorname{finsts}(H) .
\end{aligned}
$$

The second part of the proof for sequence is similar. 
Case 6: $H=\llbracket E \circledast F \circledast G \rrbracket$. Then, by the equivalence (7), we have:

$$
\begin{aligned}
\psi=\quad \circledast_{L} \cdot \operatorname{itl}(E) \wedge \text { skipstbl }\left(\circledast_{M} \cdot \operatorname{var}(F) \cup \circledast_{R} \cdot \operatorname{var}(G)\right)^{*} ; \\
\left(\circledast_{M} \cdot \operatorname{itl}(F) \wedge \text { skipstbl }\left(\circledast_{L} \cdot \operatorname{var}(E) \cup \circledast_{R} \cdot \operatorname{var}(G)\right)^{*}\right)^{*} ; \\
\circledast_{R} \cdot \operatorname{itl}(G) \wedge \text { skipstbl }\left(\circledast_{L} \cdot \operatorname{var}(E) \cup \circledast_{M} \cdot \operatorname{var}(F)\right)^{*}
\end{aligned}
$$

Hence we obtain, by $\operatorname{var}(\psi)=\circledast_{L} \cdot \operatorname{var}(\operatorname{itl}(E)) \cup \circledast_{M} \cdot \operatorname{var}(\operatorname{itl}(F)) \cup \circledast_{R} \cdot \operatorname{var}(\operatorname{itl}(G))$ as well as (applied in this order) Proposition 3.3, Proposition 3.5, the induction hypothesis, and Proposition 2.5:

$$
\begin{aligned}
\text { finsts }(\psi) & =\text { finsts }\left(\circledast_{L} \cdot \operatorname{itl}(E)\right) \cdot \text { finsts }\left(\circledast_{R} \cdot \operatorname{itl}(F)\right)^{*} \cdot \text { finsts }\left(\circledast_{M} \cdot \operatorname{itl}(G)\right) \\
& =\circledast_{L} \cdot \text { finsts }(\operatorname{itl}(E)) \cdot\left(\circledast_{R} \cdot \text { finsts }(i t l(F))\right)^{*} \cdot \circledast_{M} \cdot \text { finsts }(i t l(G)) \\
& =\circledast_{L} \cdot \text { finsts }(\operatorname{box}(E)) \cdot\left(\circledast_{R} \cdot \text { finsts }(\operatorname{box}(F))\right)^{*} \cdot \circledast_{M} \cdot \operatorname{finsts}(\operatorname{box}(G))=\text { finsts }(H) .
\end{aligned}
$$

The second part of the proof for iteration is similar.

\subsection{Translating streamlined expressions}

The result captured by Theorem 4.1 is strong as it means that the behavioural properties of non-synchronised box expressions related to the sequencing of executed actions can be re-interpreted as properties of the translated formulas, assuming that an execution of a transition is 'simulated' by a flipping of the corresponding Boolean variable. Extending such a result to streamlined expressions highlights the way in which the box expression synchronisation mechanism (through merging transitions) and the ITL synchronisation mechanism (through flipping variables in different parts of a formula) can be made to match each other.

Let $F=E$ sco $\rho$ be a streamlined box expression. Then, $\operatorname{itl}(F)$ is obtained from itl $(E)$ by replacing each occurrence of each variable $v$ by the unique variable in $\operatorname{trans}(v)^{8}$.

It is also important to stress that it is not necessary to derive box $(F)$ in order to derive the trans $(v)$ 's.

\section{Theorem 4.2. (streamlined expression)}

Let $F=E$ sco $\rho$ be a streamlined box expression. Then, sts $(\operatorname{itl}(F))=s t s(\operatorname{box}(F))$, for sts $=$ infsts or $s t s=$ finsts.

\section{Proof:}

By the definition of the itl mapping, flipping the value of any variable $v$ in itl $(E)$ is due to the (unique within it $(E)$ ) sub-formula flip $(v)$ as otherwise $v$ keeps the same value due to the presence of the skipstbl $(v)^{*}$ sub-formulas.

Suppose now that $a_{W}$ is a variable in $\operatorname{itl}(F)$ and that $a_{\pi_{1}}^{1}, \ldots, a_{\pi_{k}}^{k}$ are the variables in itl $(E)$ which in $\operatorname{itl}(F)$ are replaced by $a_{W}$. Then, by definition, $\pi_{i} \mid \pi_{j}$ for all $i \neq j$. Hence, there is a sub-formula $\varphi \wedge \varphi^{\prime}$ of $\operatorname{itl}(E)$ such that $\operatorname{var}(\varphi) \cap \operatorname{var}\left(\varphi^{\prime}\right)=\varnothing$ and, without loss of generality, $a_{\pi_{i}}^{i} \in \operatorname{var}(\varphi)$ and $a_{\pi_{j}}^{j} \in \operatorname{var}\left(\varphi^{\prime}\right)$. As this observation holds for all distinct $i$ and $j$, it follows that flipping of $a_{W}$ in $\operatorname{itl}(F)$ must be 'agreed upon' by all the sub-formulas flip $\left(a_{W}\right)$, each resulting from a replacement of some $a_{\pi_{j}}^{i}$ by $a_{W}$. The result then follows from sts $(\mathrm{itl}(E))=s t s(\operatorname{box}(E))$ (see Theorem 4.1) and Proposition 2.6.

\footnotetext{
${ }^{8}$ Recall trans $(t)$ was introduced in Section 2.6.
} 
In terms of complexity, the size of $\operatorname{itl}(F)$ is of the same order as that $\operatorname{itl}(E)$, and so $|\operatorname{itl}(F)| \in$ $O\left(|F|^{2}\right)$ which, again, compares very favourably with $|\operatorname{box}(F)|$ as $\left|\operatorname{box}(F)^{\top}\right| \in O\left(2^{|E|}\right)$. Moreover, the number variables in $\operatorname{itl}(F)$ is the same as the number of transitions in box $(F)$.

\subsection{Translating general expressions}

Suppose now that $F=E \mathrm{sco} \rho$ is an arbitrary synchronised expression. Given Proposition 2.7, we could now simply take the streamlined expression $\operatorname{st}(F)$ defined in Section 2.6 and, after consistently renaming variables according to the bijection $\lambda$ defined in Section 2.6, derive $\operatorname{itl}(\operatorname{stl}(F))$ and obtain a generalised version of Theorem 4.2.

Applying our previous complexity estimates, we would get $|\operatorname{itl}(\operatorname{stl}(F))| \in O\left(\left(|E|+|\operatorname{box}(E)|^{\mathrm{F}}\right)^{2}\right)$. Therefore, $\operatorname{itl}(\operatorname{stl}(F))$ would be of similar size as $\operatorname{box}(E)$. This, however, changes radically if we assumed that, e.g., only binary synchronisations are allowed and a given pair of synchronised actions always yields the same action label, ${ }^{9}$ and so $|\operatorname{box}(E)|^{\mathrm{F}} \in O\left(|E|^{2}\right)$. Then we would have $|\operatorname{itl}(\operatorname{stl}(F))| \in$ $O\left(|E|^{4}\right)$ whereas $|\operatorname{box}(E)|$ would belong to $O\left(2^{|E|}\right) .{ }^{10}$ Moreover, as we already argued, it is not necessary to construct box $(F)$ in order to derive $\operatorname{itl}(\operatorname{stl}(F))$.

As an alternative, we may proceed without pre-processing and conservatively extend the translation defined for non-synchronised expressions. More precisely, for any synchronised expression $F=E$ sco $\rho$, we construct $\operatorname{itl}(F)$ directly from itl $(E)$ by replacing each flip $(t)$ by flip $(\operatorname{trans}(t))$, and each skipstbl $(V)$ by skipstbl $(\bigcup \operatorname{trans}(V))$. The size of itl $(F)$ would be similar as that of itl(stl $(F))$.

\section{Theorem 4.3. (synchronised expression)}

Let $F=E$ sco $\rho$ be a synchronised box expression. Then, sts $(\operatorname{itl}(F))=\operatorname{sts}(\operatorname{box}(F))$, where sts $=$ infsts or $s t s=$ finsts.

\section{Proof:}

Let us consider the streamlined expression $\operatorname{stl}(E \operatorname{sco} \rho)=E^{\prime}$ sco $\rho^{\prime}$, as defined in Section 2.6, and $\varphi=\operatorname{itl}\left(E^{\prime} \operatorname{sco} \rho^{\prime}\right)$ as defined for streamlined expression. Then $\operatorname{itl}(E \operatorname{sco} \rho)$ and $\varphi$ are equivalent after applying a consistent renaming of variables given by the bijection $\lambda$ defined in Section 2.6. Now, we observe that if trans $\left(a_{\pi}\right)=\left\{b_{W_{1}}^{1}, \ldots, b_{W_{m}}^{m}\right\}$ and $m \geq 2$, then the sub-formula fs $\left(a_{\pi} \mid \varnothing\right)$ in itl $(E)$ is transformed into $\mathrm{fs}\left(\operatorname{trans}\left(a_{\pi}\right) \mid \varnothing\right)$ within itl $(F)$. This is equivalent to

$$
\bigvee_{1 \leq i \leq m} \mathrm{fs}\left(b_{W_{i}}^{i} \mid b_{W_{1}}^{1}, \ldots, b_{W_{i-1}}^{i-1}, b_{W_{i+1}}^{i+1}, \ldots, b_{W_{m}}^{m}\right),
$$

which in turn can be shown to be equivalent (after taking into account the correspondence given by $\lambda$ ) to itl $\left(b_{W_{1}}^{1} \square \ldots \square b_{W_{m}}^{m}\right)$. Hence, the result follows from Theorem 4.2 and Propositions 2.7 and 3.4.

To conclude, we have demonstrated that it is possible to associate in a computationally efficient way a semantically equivalent ITL formula with any box expression considered in this paper.

\footnotetext{
${ }^{9}$ Such an assumption is usually made, e.g., by process algebras.

${ }^{10}$ Take, for every $n \geq 1, F_{n}=\underbrace{(\text { stop } \| \text { stop }) \square \ldots \square(\text { stop } \| \text { stop })}_{n \text { times }})$ sco $\varnothing$. Then $\left|F_{n}\right|=2 \cdot n$ and $\mid$ box $\left(F_{n}\right) \mid=2^{n+1}$.
} 


\section{Examples}

We will now present examples illustrating the translation of box expressions into logical formulas.

\subsection{Synchronisation and restriction}
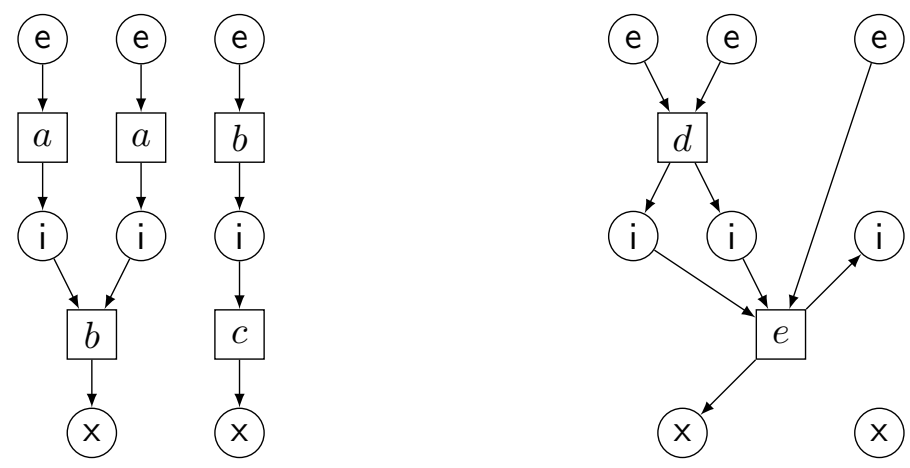

Figure 5. Boxes of $E=((a \| a) ; b) \|(b ; c)$ and $F=E \operatorname{sco}\{a a \mapsto d, b b \mapsto e\}$.

To illustrate action restriction as well as internal and external synchronisations, we consider a streamlined expression $F=E \operatorname{sco} \rho$, where

$$
E=((a \| a) ; b) \|(b ; c) \text { and } \rho=\{a a \mapsto d, b b \mapsto e\} .
$$

The corresponding boxes are shown in Figure 5. In the translation, we first derive:

$$
\begin{aligned}
& \operatorname{itl}(E)=\left(\left(\mathrm{fs}\left(a_{\left\|_{L} ; ;_{L}\right\|_{L}} \mid b_{\|_{L}} ;_{R}\right) \wedge \mathrm{fs}\left(a_{\left\|_{L} ;_{L}\right\|_{R}} \mid b_{\|_{L}} ;_{R}\right)\right) ; \mathrm{fs}\left(b_{\|_{L}} ;_{R} \mid a_{\|_{L}} ;_{L}\left\|_{L}, a_{\|_{L}} ;_{L}\right\|_{R}\right)\right) \\
& \wedge\left(\mathrm{fs}\left(b_{\| R} ; L \mid c_{\| R} ; R\right) ; \mathrm{fs}\left(c_{\| R} ; R \mid b_{\| R} ; L\right)\right) \text {. }
\end{aligned}
$$

To prepare for applying scoping, we derive

$$
\begin{aligned}
& \operatorname{trans}\left(a_{\|_{L} ; L} ;_{L}\right)=\operatorname{trans}\left(a_{\|_{L} ; L} ;_{L}\right)=\left\{d_{\|_{L} ; L} ;_{L},\left\|_{L} ;_{L}\right\|_{R}\right\}=\{\alpha\} \\
& \operatorname{trans}\left(b_{\|_{L} ; R}\right)=\operatorname{trans}\left(b_{\|_{R} ; L}\right)=\left\{e_{\left\|_{L} ; R,\right\|_{R} ; L}\right\}=\{\beta\} \\
& \operatorname{trans}\left(c_{\|_{R} ; R}\right)=\varnothing
\end{aligned}
$$

which after simplifications leads to

$$
\begin{array}{rlrl}
\operatorname{itl}(F) & =((\mathrm{fs}(\alpha \mid \beta) \wedge \mathrm{fs}(\alpha \mid \beta)) ; \mathrm{fs}(\beta \mid \alpha)) & \wedge(\mathrm{fs}(\beta \mid \varnothing) ; \operatorname{infstbl}(\beta)) \\
& =\quad(\mathrm{fs}(\alpha \mid \beta) ; \mathrm{fs}(\beta \mid \alpha)) & \wedge(\mathrm{fs}(\beta \mid \varnothing) ; \operatorname{infstbl}(\beta)) \\
& =\mathrm{fs}(\alpha \mid \beta) ; \mathrm{fs}(\beta \mid \alpha) ; \operatorname{infstbl}(\alpha, \beta) .
\end{array}
$$

Thus $\operatorname{itl}(F)$ is satisfied over an interval provided that the latter can be split into three successive subintervals (the first two being finite and the third one infinite) overlapping on single states so that the 
variables $\alpha$ and $\beta$ are kept unchanged, except for one flipping of $\alpha$ within the first interval, and one flipping of $\beta$ in the second interval (see (4) for the definitions of $\mathrm{fs}(\alpha \mid \beta), \mathrm{fs}(\beta \mid \alpha)$, and infstbl $(\alpha, \beta)$ ). Note that finsts $(F)=\varnothing$ and $\operatorname{infsts}(F)=\varnothing^{\omega} \cup \varnothing^{*} \cdot\{\{\alpha\}\} \cdot \varnothing^{\omega} \cup \varnothing^{*} \cdot\{\{\alpha\}\} \cdot \varnothing^{*} \cdot\{\{\beta\}\} \cdot \varnothing^{\omega}$.

\subsection{Parallel composition and choice}

To illustrate choice and parallel composition, we consider a streamlined expression $F=E$ sco $\rho$, where

$$
E=(a \square b) \| b \quad \text { and } \quad \rho=\{a \mapsto a, b b \mapsto b\} .
$$

The corresponding boxes are shown in Figure 6. In the translation, we first derive

$$
\operatorname{itl}(E)=\left(\mathrm{fs}\left(a_{\|\|_{L} \square_{L}} \mid b_{\|_{L}} \square_{R}\right) \vee \mathrm{fs}\left(b_{\|_{L}} \square_{R} \mid a_{\|_{L} \square_{L}}\right)\right) \wedge \mathrm{fs}\left(b_{\|_{R}} \mid \varnothing\right) .
$$

To prepare for applying scoping, we derive:

$$
\begin{aligned}
\operatorname{trans}\left(a_{\| L} \square_{L}\right) & =\left\{a_{\| L} \square_{L}\right\}=\{\alpha\} \\
\operatorname{trans}\left(b_{\| L} \square_{R}\right)=\operatorname{trans}\left(b_{\| R}\right) & =\left\{b_{\| L} \square_{R}, \|_{R}\right\}=\{\beta\}
\end{aligned}
$$

which (after eliminating true in conjunctions) leads to

$$
\operatorname{itl}(F)=(\mathrm{fs}(\alpha \mid \beta) \vee \mathrm{fs}(\beta \mid \alpha)) \wedge \mathrm{fs}(\beta \mid \varnothing) \equiv(\mathrm{fs}(\alpha \mid \beta) ; \operatorname{infstbl}(\alpha, \beta)) \vee \mathrm{fs}(\beta \mid \alpha) .
$$

Hence finsts $(F)=\varnothing^{*} \cdot\{\{\beta\}\} \cdot \varnothing^{*}$ and infsts $(F)=\varnothing^{\omega} \cup \varnothing^{*} \cdot\{\{\alpha\},\{\beta\}\} \cdot \varnothing^{\omega}$.
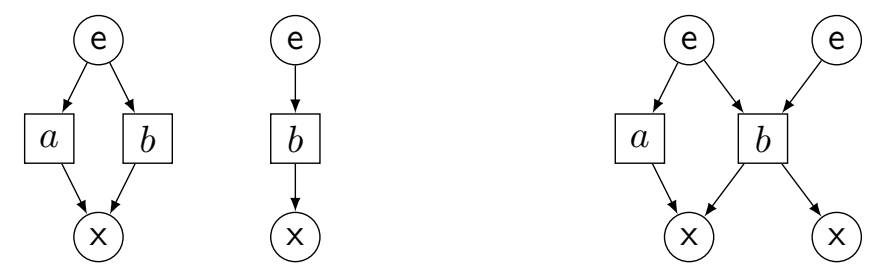

Figure 6. Boxes of $E=(a \square b) \| b$ and $F=E \operatorname{sco}\{a \mapsto a, b b \mapsto b\}$.

\subsection{Iteration and scoping}

To illustrate synchronisation inside iteration, we consider a non-streamlined expression $F=E$ sco $\rho$, where

$$
E=\llbracket a \circledast(b \| c) \circledast d \rrbracket \quad \text { and } \quad \rho=\{a \mapsto a, b c \mapsto a, c \mapsto e\} .
$$

The boxes corresponding to $E$ and $F$ are shown in Figure 4. We first derive

$$
\operatorname{itl}(E)=\operatorname{fs}\left(a_{\circledast_{L}} \mid b_{\circledast_{M}{ }^{\prime} L}, c_{\circledast_{M} \|_{R}}, d_{\circledast_{R}}\right) ;\left(\begin{array}{c}
\mathrm{fs}\left(b_{\circledast_{M} \|_{L}} \mid a_{\circledast_{L}}, d_{\circledast_{R}}\right) \\
\wedge \\
\mathrm{fs}\left(c_{\circledast_{M} \|_{R}} \mid a_{\circledast_{L}}, d_{\circledast_{R}}\right)
\end{array}\right)^{*} ; \mathrm{fs}\left(d_{\circledast_{R}} \mid a_{\circledast_{L}}, b_{\circledast_{M} \|_{L}}, c_{\circledast_{M} \|_{R}}\right) .
$$


To prepare for applying scoping, we derive

$$
\begin{array}{rll}
\operatorname{trans}\left(a_{\circledast_{L}}\right) & =\left\{a_{\circledast_{L}}\right\} & =\{\gamma\} \\
\operatorname{trans}\left(d_{\circledast_{R}}\right) & =\varnothing & =\{\alpha\} \\
\operatorname{trans}\left(b_{\circledast_{M}{ }^{\prime} L}\right) & =\left\{a_{\circledast_{M}\left\|_{L}, \circledast_{M}\right\|_{R}}\right\} & =\{\alpha, \zeta\} \\
\operatorname{trans}\left(c_{\circledast_{M}{ }^{\prime} R}\right) & =\left\{a_{\circledast_{M}{ }^{\prime} L}, \circledast_{M{ }^{\prime} R}, e_{\circledast_{M} \|_{R}}\right\} & =\{
\end{array}
$$

which leads to

$$
\begin{aligned}
\operatorname{itl}(F) & =\mathrm{fs}(\gamma \mid \alpha, \zeta) ;(\mathrm{fs}(\alpha \mid \gamma) \wedge \mathrm{fs}(\alpha, \zeta \mid \gamma))^{*} ; \operatorname{infstbl}(\gamma, \alpha, \zeta) \\
& \equiv \mathrm{fs}(\gamma \mid \alpha, \zeta) ;((\mathrm{fs}(\alpha \mid \gamma) \wedge \mathrm{fs}(\alpha \mid \gamma, \zeta)) \vee(\mathrm{fs}(\alpha \mid \gamma) \wedge \mathrm{fs}(\zeta \mid \gamma, \alpha)))^{*} ; \operatorname{infstbl}(\gamma, \alpha, \zeta) \\
& \equiv \mathrm{fs}(\gamma \mid \alpha, \zeta) ;(\mathrm{fs}(\alpha \mid \gamma, \zeta) \vee(\mathrm{fs}(\zeta \mid \gamma, \alpha) ; \operatorname{infstbl}(\gamma, \alpha, \zeta)))^{*} ; \text { infstbl }(\gamma, \alpha, \zeta) .
\end{aligned}
$$

Hence infsts $(F)=\varnothing^{\omega} \cup \varnothing^{*} \cdot\{\{\gamma\}\} \cdot\left\{\varnothing^{\omega} \cup\left(\varnothing^{*} \cdot\{\{\alpha\}\}\right)^{*} \cdot \varnothing^{\omega} \cup\left(\varnothing^{*} \cdot\{\{\alpha\}\}\right)^{*} \cdot \varnothing^{*} \cdot\{\{\zeta\}\} \cdot \varnothing^{\omega}\right\}$ and finsts $(F)=\varnothing$.

\subsection{Producer/consumers system}

Consider BA expressions $G_{n}(n \geq 1)$, modelling a producer $P_{n}$ working in parallel with $n$ consumer processes $C_{1}, \ldots, C_{n}$. After starting up using action $a$, the producer repeatedly performs a local action $b$ followed by a parallel execution of communication actions $c_{1}, \ldots, c_{n}$, each cycle being finished by the execution of action $d$, and then a new cycle is started by executing action $e$. A consumer process $C_{i}$, after a start up action $a_{i}$, also executes an indefinite loop executing action $c_{i}$ and ending each cycle with $f_{i}$. The definitions of these expressions are as follows:

$$
\begin{aligned}
C_{i} & =\llbracket a_{i} \circledast c_{i} ; f_{i} \circledast \text { stop } \rrbracket \\
P_{n} & =\llbracket a \circledast b ;\left(c_{1}\|\ldots\| c_{n}\right) ; d ; e \circledast \text { stop } \rrbracket \\
P C_{n} & =\left(C_{1}\|\ldots\| C_{n} \| P_{n}\right) \operatorname{sco} \rho
\end{aligned}
$$

where

$$
\rho=\left\{\begin{array}{rlrlrl}
c_{1} c_{1} & \mapsto \tau_{1} & \ldots & c_{n} c_{n} \mapsto \tau_{n} & a \mapsto a \\
a_{1} & \mapsto a_{1} & \ldots & a_{n} \mapsto a_{n} & d \mapsto d \quad b \mapsto b \\
f_{1} \mapsto f_{1} & \ldots & f_{n} \mapsto f_{n} & e \mapsto e
\end{array}\right\}
$$

Figure 7 depicts the boxes of $C_{i}$ and $P_{n}$. Moreover, Figure 8 shows the box corresponding to $P C_{2}$ together with the initial marking. The corresponding ITL formula is shown below (for brevity, we only indicate variables which flip their values, but even then we omit the subscripts, and show only the labels of transitions corresponding to these variables):

$$
\begin{aligned}
\operatorname{itl}\left(P C_{n}\right)= & \left(\mathrm{fs}\left(a_{1} \mid\right) ;\left(\mathrm{fs}\left(\tau_{1} \mid\right) ; \mathrm{fs}\left(f_{1} \mid\right)\right)^{*} ; \operatorname{infstbl}()\right) \wedge \cdots \wedge \\
& \left(\mathrm{fs}\left(a_{n} \mid\right) ;\left(\mathrm{fs}\left(\tau_{n} \mid\right) ; \mathrm{fs}\left(f_{n} \mid\right)\right)^{*} ; \operatorname{infstbl}()\right) \wedge \\
& \left(\mathrm{fs}(a \mid) ;\left(\mathrm{fs}(b \mid) ;\left(\mathrm{fs}\left(\tau_{1} \mid\right) \wedge \cdots \wedge \mathrm{fs}\left(\tau_{n} \mid\right)\right) ; \mathrm{fs}(d \mid) ; \mathrm{fs}(e \mid)\right)^{*} ; \operatorname{infstbl}()\right) .
\end{aligned}
$$



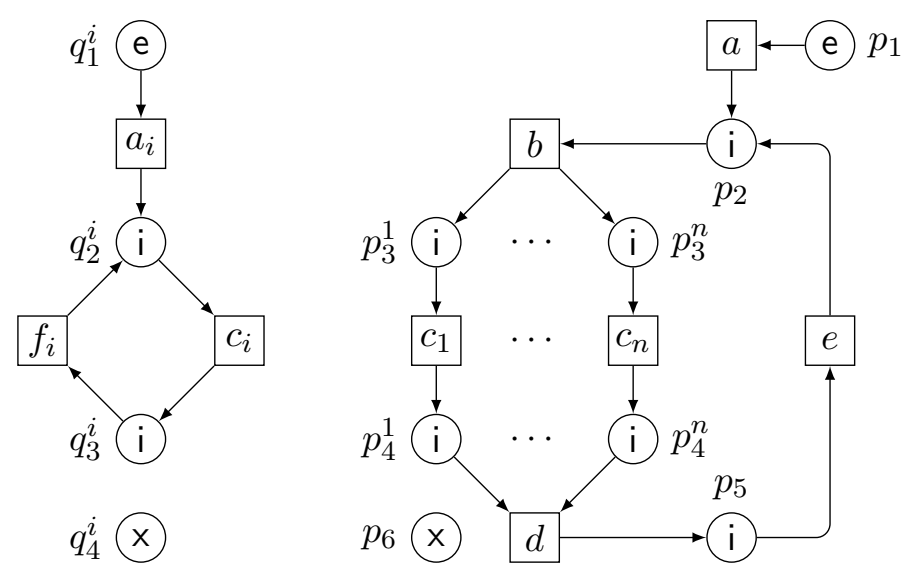

Figure 7. The boxes of the consumers $C_{i}$ and the producer $P_{n}$.

\section{Conclusions}

In the past, logics have been mainly used for expressing correctness properties of systems specified using Petri nets [36]. When it comes to the intrinsic semantic relationship between logics and Petri nets, we feel that the work on the connections between linear logic [37] and Place Transition nets has been the closest one. However, the main concern there was the handling of multiple token occurrences in net places whereas here nets can hold at most two tokens in a single place. Another way in which logics and Petri nets are related is reported in [38], which provided a characterisation of Petri net languages in terms of second-order logical formulas.

The results presented in this paper demonstrate that one can define a translation from box expressions to ITL with equivalent behaviour. We also show that the complexity of the proposed translation compares favourably with the complexity of the translation from box expressions to boxes.

It is therefore important to further investigate the extent to which the established connection between BA and ITL could be generalised and exploited. In particular, we plan to investigate what is the subset of ITL which can be translated into BA. We do not expect that such a translation will be easy for the full ITL for at least two reasons. The first is that the projection operator prj of ITL does not have an equivalent in BA and so incorporating it would call for new constructs at the syntactical and semantical levels. The second reason is that ITL allows one to specify actions happening simultaneously, e.g., , as in flip $(v) \wedge f$ lip $(w)$. This is akin to executing the step $\{v, w\}$ without being able to execute $\{v\}\{w\}$ or $\{w\}\{v\}$, i.e., $v$ and $w$ must be executed synchronously and without delay. Such a behaviour cannot be reproduced in the standard Petri nets (and also boxes) as they are inherently asynchronous models. The required effect could perhaps be achieved by using the maximally concurrent execution rule, but this would fundamentally change the net model. In fact, we feel that in logical formulas translatable to box expressions all actions should be fully asynchronous, using $\mathrm{fs}(v \mid \varnothing)$ rather than $v$ in ITL syntax. However, more investigation needs to be conducted in order, in particular, to generate a suitable scoping set from a give ITL formula. 


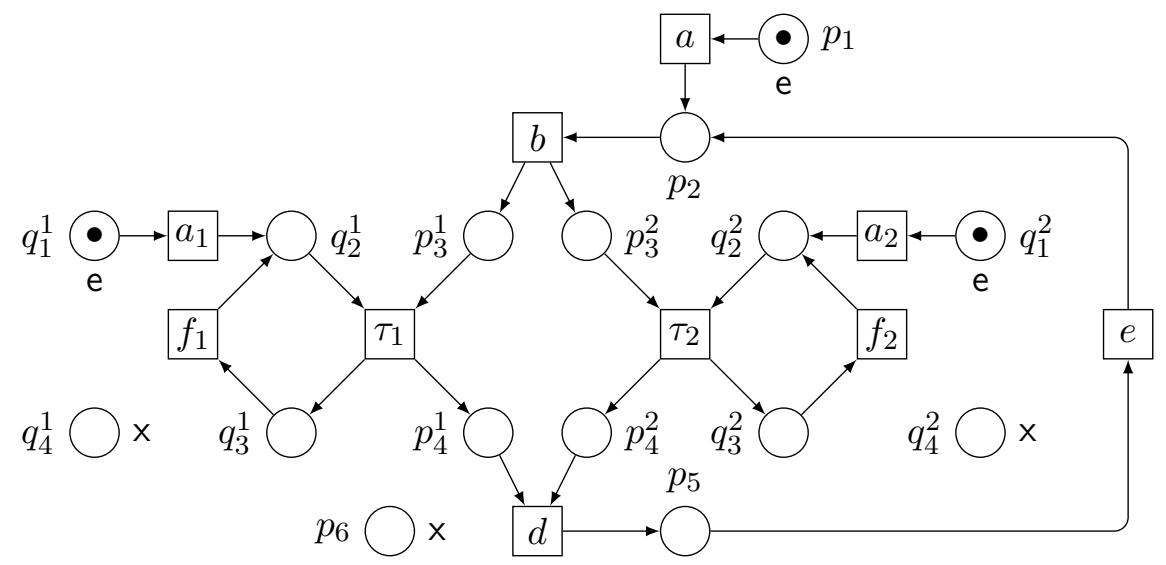

Figure 8. Initially marked box of $\mathrm{PC}_{2}$.

A long-term goal is the development of a hybrid verification methodology combining ITL and BA techniques. For example, sequential algorithms and infinite data structures could be treated by ITL techniques [39, 40, 41, 30], while intensive parallel or communicating aspects of systems could be treated by net unfoldings [42, 43] or other Petri net techniques [44].

\section{Acknowledgement}

This research was supported by the 973 Program Grant 2010CB328102, NSFC Grant 61133001, ANR SYNBIOTIC and EPSRC UNCOVER project.

\section{References}

[1] Goranko V, Montanari A, Sciavicco G. A Road Map of Interval Temporal Logics and Duration Calculi. Journal of Applied Non-Classical Logics, 2004. 14(1-2):9-54. doi:10.3166/jancl.14.9-54. URL https://doi.org/10.3166/jancl.14.9-54.

[2] Emerson EA. Temporal and Modal Logic. In: Handbook of Theoretical Computer Science, Volume B: Formal Models and Sematics (B), pp. 995-1072. MIT Press Cambridge, 1990.

[3] Manna Z, Pnueli A. Verification of Concurrent Programs: Temporal Proof Principles. In: Kozen D (ed.), Logics of Programs, Workshop, Yorktown Heights, New York, USA, May 1981, volume 131 of Lecture Notes in Computer Science. Springer. ISBN 3-540-11212-X, 1981 pp. 200-252. doi: 10.1007/BFb0025785. URL https://doi .org/10.1007/BFb0025785.

[4] Moszkowski BC, Guelev DP, Leucker M. Guest editors' preface to special issue on interval temporal logics. Ann. Math. Artif. Intell., 2014. 71(1-3):1-9. doi:10.1007/s10472-014-9417-7. URL https://doi.org/10.1007/s10472-014-9417-7.

[5] Desel J, Juhás G. "What Is a Petri Net?". In: Ehrig H, Juhás G, Padberg J, Rozenberg G (eds.), Unifying Petri Nets, Advances in Petri Nets, volume 2128 of Lecture Notes in Computer 
Science. Springer. ISBN 3-540-43067-9, 2001 pp. 1-25. doi:10.1007/3-540-45541-8_1. URL https://doi.org/10.1007/3-540-45541-8_1.

[6] Peterka G, Murata T. Proof Procedure and Answer Extraction in Petri Net Model of Logic Programs. IEEE Trans. Software Eng., 1989. 15(2):209-217. doi:10.1109/32.21746. URL https://doi.org/10.1109/32.21746.

[7] Suárez MS, Teruel E, Colom JM. Linear Algebraic and Linear Programming Techniques for the Analysis of Place or Transition Net Systems. In: Reisig W, Rozenberg G (eds.), Lectures on Petri Nets I: Basic Models, Advances in Petri Nets, the volumes are based on the Advanced Course on Petri Nets, held in Dagstuhl, September 1996, volume 1491 of Lecture Notes in Computer Science. Springer. ISBN 3-540-65306-6, 1996 pp. 309-373. doi:10.1007/3-540-65306-6_19. URL https://doi.org/10.1007/3-540-65306-6_19.

[8] McMillan KL. A Technique of State Space Search Based on Unfolding. Formal Methods in System Design, 1995. 6(1):45-65. doi:10.1007/BF01384314. URL https://doi .org/10.1007/BF01384314.

[9] Valmari A. Stubborn sets for reduced state space generation. In: Rozenberg G (ed.), Advances in Petri Nets 1990 [10th International Conference on Applications and Theory of Petri Nets, Bonn, Germany, June 1989, Proceedings], volume 483 of Lecture Notes in Computer Science. Springer. ISBN 3-540-53863-1, 1989 pp. 491-515. doi:10.1007/3-540-53863-1_36. URL https ://doi .org/10.1007/3-540-53863-1_36.

[10] Duan Z, Klaudel H, Koutny M. ITL semantics of composite Petri nets. $J$. Log. Algebr. Program., 2013. 82(2):95-110. doi:10.1016/j.jlap.2012.12.001. URL https://doi.org/10.1016/j.jlap.2012.12.001.

[11] Moszkowski BC. Compositional reasoning about projected and infinite time. In: 1st IEEE International Conference on Engineering of Complex Computer Systems (ICECCS '95), November 6-10, 1995, Fort Lauderdale, Florida, USA. IEEE Computer Society. ISBN 0-8186-7123-8, 1995 pp. 238-245. doi: 10.1109/ICECCS.1995.479336. URL https : //doi .org/10.1109/ICECCS . 1995. 479336.

[12] and Zohar Manna. Reasoning in Interval Temporal Logic. In: Clarke EM, Kozen D (eds.), Logics of Programs, Workshop, Carnegie Mellon University, Pittsburgh, PA, USA, June 6-8, 1983, Proceedings, volume 164 of Lecture Notes in Computer Science. Springer. ISBN 3-540-12896-4, 1983 pp. 371-382. doi:10.1007/3-540-12896-4_374. URL https ://doi.org/10.1007/3-540-12896-4_374.

[13] Best E, Devillers R, Koutny M. Petri Net Algebra. Monographs in Theoretical Computer Science. Springer, 2001.

[14] Best E, Devillers RR, Hall JG. The box calculus: a new causal algebra with multi-label communication. In: Rozenberg G (ed.), Advances in Petri Nets 1992, The DEMON Project, volume 609 of Lecture Notes in Computer Science, pp. 21-69. Springer. ISBN 3-540-55610-9, 1992. doi:10.1007/3-540-55610-9_167. URL https://doi.org/10.1007/3-540-55610-9_167.

[15] Milner R. A Calculus of Communicating Systems. Springer, 1980.

[16] CARHoare. Communicating Sequential Processes. Prentice-Hall, 1985.

[17] Best E, Fraczak W, Hopkins RP, Klaudel H, Pelz E. M-Nets: An Algebra of High-Level Petri Nets, with an Application to the Semantics of Concurrent Programming Languages. Acta Inf., 1998. 35(10):813-857. doi:10.1007/s002360050144. URL https://doi.org/10.1007/s002360050144.

[18] Macià H, Ruiz VV, Cuartero F, de Frutos-Escrig D. A congruence relation for sPBC. Formal Methods in System Design, 2008. 32(2):85-128. doi:10.1007/s10703-007-0045-2. URL https://doi.org/10.1007/s10703-007-0045-2. 
[19] Goranko V, Montanari A. Foreword to Special Issue on Interval Temporal Logics and Duration Calculi. Journal of Applied Non-Classical Logics, 2004. 14(1-2):7-8.

[20] Bäumler S, Schellhorn G, Tofan B, Reif W. Proving linearizability with temporal logic. Formal Asp. Comput., 2011. 23(1):91-112. doi:10.1007/s00165-009-0130-y. URL https://doi.org/10.1007/s00165-009-0130-y.

[21] Halpern JY, Shoham Y. A Propositional Modal Logic of Time Intervals. J. ACM, 1991. 38(4):935-962. doi:10.1145/115234.115351. URL https://doi.org/10.1145/115234.115351.

[22] Venema Y. A Modal Logic for Chopping Intervals. J. Log. Comput., 1991. 1(4):453-476. doi: 10.1093/logcom/1.4.453. URL https://doi.org/10.1093/logcom/1.4.453.

[23] Allen JF. Maintaining Knowledge about Temporal Intervals. Commun. ACM, 1983. 26(11):832-843. doi:10.1145/182.358434. URL http://doi.acm.org/10.1145/182.358434.

[24] Bozzelli L, Molinari A, Montanari A, Peron A, Sala P. Interval vs. Point Temporal Logic Model Checking: An Expressiveness Comparison. ACM Trans. Comput. Log., 2019. 20(1):4:1-4:31. doi:10.1145/3281028. URL https://doi.org/10.1145/3281028.

[25] Lomuscio A, Michaliszyn J. An Epistemic Halpern-Shoham Logic. In: Rossi F (ed.), IJCAI 2013, Proceedings of the 23rd International Joint Conference on Artificial Intelligence, Beijing, China, August 3-9, 2013. IJCAI/AAAI. ISBN 978-1-57735-633-2, 2013 pp. 1010-1016. URL http://www . aaai .org/ocs/index.php/IJCAI/IJCAI13/paper/view/6632.

[26] Lomuscio AR, Michaliszyn J. Decidability of model checking multi-agent systems against a class of EHS specifications. In: Schaub T, Friedrich G, O'Sullivan B (eds.), ECAI 2014 - 21st European Conference on Artificial Intelligence, 18-22 August 2014, Prague, Czech Republic - Including Prestigious Applications of Intelligent Systems (PAIS 2014), volume 263 of Frontiers in Artificial Intelligence and Applications. IOS Press. ISBN 978-1-61499-418-3, 2014 pp. 543-548. doi:10.3233/978-1-61499-419-0-543. URL https://doi.org/10.3233/978-1-61499-419-0-543.

[27] Montanari A, Murano A, Perelli G, Peron A. Checking Interval Properties of Computations. In: Cesta A, Combi C, Laroussinie F (eds.), 21st International Symposium on Temporal Representation and Reasoning, TIME 2014, Verona, Italy, September 8-10, 2014. IEEE Computer Society. ISBN 978-1-4799-4228-2, 2014 pp. 59-68. doi:10.1109/TIME.2014.24. URL https://doi.org/10.1109/TIME. 2014.24.

[28] Molinari A, Montanari A, Murano A, Perelli G, Peron A. Checking interval properties of computations. Acta Inf., 2016. 53(6-8):587-619. doi:10.1007/s00236-015-0250-1. URL https://doi.org/10.1007/s00236-015-0250-1.

[29] http://www.antonio-cau.co.uk/ITL/.

[30] Moszkowski BC. Executing Temporal Logic Programs. Cambridge University Press, 1986.

[31] IEEE: Standard for the Functional Verification Language e, Standard 1647-2011. ANSI/IEEE, New York, 2011.

[32] Chaochen Z, Hoare CAR, Ravn AP. A Calculus of Durations. Inf. Process. Lett., 1991. 40(5):269-276. doi:10.1016/0020-0190(91)90122-X. URL https : //doi .org/10.1016/0020-0190(91)90122-X.

[33] Linker S, Hilscher M. Proof Theory of a Multi-Lane Spatial Logic. Logical Methods in Computer Science, 2015. 11(3). doi:10.2168/LMCS-11(3:4)2015. URL https://doi .org/10.2168/LMCS-11 (3:4) 2015. 
[34] Klaudel H, Koutny M, Moszkowski BC. From Petri Nets with Shared Variables to ITL. In: Desel J, Yakovlev A (eds.), 16th International Conference on Application of Concurrency to System Design, ACSD 2016, Torun, Poland, June 19-24, 2016. IEEE Computer Society. ISBN 978-1-5090-2589-3, 2016 pp. $11-$ 18. doi:10.1109/ACSD.2016.12. URL https : //doi .org/10.1109/ACSD . 2016.12.

[35] Klaudel H, Koutny M, Duan Z. Interval Temporal Logic Semantics of Box Algebra. In: Dediu A, Martín-Vide C, Sierra-Rodríguez JL, Truthe B (eds.), Language and Automata Theory and Applications - 8th International Conference, LATA 2014, Madrid, Spain, March 10-14, 2014. Proceedings, volume 8370 of Lecture Notes in Computer Science. Springer. ISBN 978-3-319-04920-5, 2014 pp. 441-452. doi:10.1007/978-3-319-04921-2_36. URL https ://doi.org/10.1007/978-3-319-04921-2_36.

[36] Esparza J, Nielsen M. Decidability Issues for Petri Nets - a survey. Elektronische Informationsverarbeitung und Kybernetik, 1994. 30(3):143-160.

[37] Girard J. Linear Logic. Theor. Comput. Sci., 1987. 50:1-102. doi:10.1016/0304-3975(87)90045-4. URL https://doi.org/10.1016/0304-3975(87)90045-4.

[38] Parigot M, Pelz E. A Logical Approach of Petri Net Languages. Theor. Comput. Sci., 1985. 39:155-169. doi:10.1016/0304-3975(85)90136-7. URL https : //doi .org/10.1016/0304-3975 (85) 90136-7.

[39] Cau A, Janicke H, Moszkowski BC. Verification and enforcement of access control policies. Formal Methods in System Design, 2013. 43(3):450-492. doi:10.1007/s10703-013-0187-3. URL https://doi.org/10.1007/s10703-013-0187-3.

[40] Cau A, Zedan H. Refining Interval Temporal Logic Specifications. In: Bertran M, Rus T (eds.), Transformation-Based Reactive Systems Development, 4th International AMAST Workshop on RealTime Systems and Concurrent and Distributed Software, ARTS'97, Palma, Mallorca, Spain, May 21-23, 1997, Proceedings, volume 1231 of Lecture Notes in Computer Science. Springer. ISBN 3-540-63010-4, 1997 pp. 79-94. doi:10.1007/3-540-63010-4_6. URL https ://doi.org/10.1007/3-540-63010-4_6.

[41] Janicke H, Cau A, Siewe F, Zedan H. Dynamic Access Control Policies: Specification and Verification. Comput. J., 2013. 56(4):440-463. doi:10.1093/comjnl/bxs102. URL https://doi.org/10.1093/comjnl/bxs102.

[42] Esparza J, Römer S, Vogler W. An Improvement of McMillan’s Unfolding Algorithm. In: Margaria T, Steffen B (eds.), Tools and Algorithms for Construction and Analysis of Systems, Second International Workshop, TACAS '96, Passau, Germany, March 27-29, 1996, Proceedings, volume 1055 of Lecture Notes in Computer Science. Springer. ISBN 3-540-61042-1, 1996 pp. 87-106. doi:10.1007/3-540-61042-1_40. URL https://doi.org/10.1007/3-540-61042-1_40.

[43] Khomenko V, Koutny M. Towards an Efficient Algorithm for Unfolding Petri Nets. In: Larsen KG, Nielsen M (eds.), CONCUR 2001 - Concurrency Theory, 12th International Conference, Aalborg, Denmark, August 20-25, 2001, Proceedings, volume 2154 of Lecture Notes in Computer Science. Springer. ISBN 3-540-42497-0, 2001 pp. 366-380. doi:10.1007/3-540-44685-0_25. URL https://doi.org/10.1007/3-540-44685-0_25.

[44] http://www . informatik.uni-hamburg.de/TGI/PetriNets/tools/. 